\title{
UÁM
}

Universidad Autónoma

de Madrid

\section{Biblos-e Archè}

Repositorio Institucional de la Universidad Autónoma de Madrid https://repositorio.uam.es

Esta es la versión de autor del artículo publicado en:

This is an author produced version of a paper published in:

The Journal of Physical Chemistry C 124.18 (2020): 9924 - 9939

DOI: https://doi.org/10.1021/acs.jpcc.0c00729

Copyright: (c) 2020 American Chemical Society

El acceso a la versión del editor puede requerir la suscripción del recurso Access to the published version may require subscription 


\section{Revealing the Interplay Between Covalent and}

\section{Non-covalent Interactions Driving the}

\section{Adsorption of Monosubstituted Thiourea}

\section{Derivatives on the $A u(111)$ Surface}

Ransel Barzaga, ${ }^{\dagger}+$ Mayra P. Hernández, ${ }^{\dagger}$ Fernando Aguilar-Galindo, ${ }^{\ddagger}$ and Sergio

$$
\text { Díaz-Tendero*, }+,,, \|
$$

$†$ Instituto de Ciencia y Tecnología de Materiales, Universidad de La Habana, 10700 La

$$
\text { Habana, Cuba }
$$

$\ddagger$ Departmento de Química, Módulo 13, Universidad Autónoma de Madrid, 28049 Madrid,

$$
\text { Spain }
$$

\Current address: Donostia International Physics Center (DIPC), Paseo Manuel de Lardizabal 4, Donostia-San Sebastián, E-20018, Spain

$\S$ Condensed Matter Physics Center (IFIMAC)- Universidad Autónoma de Madrid - 28049

$$
\text { Madrid - Spain }
$$

|| Institute for Advanced Research in Chemical Science (IAdChem), Universidad Autónoma de Madrid, 28049 Madrid, Spain

E-mail: sergio.diaztendero@uam.es 


\begin{abstract}
This work reports a detailed study of the adsorption of thiourea and seven monosubstituted thiourea derivatives on the $\mathrm{Au}(111)$ surface based on self-consistent periodic density functional theory (DFT) calculations. We included in our analysis the most stable cis and trans configurations for each considered monosubstituted derivative. Different functional groups have been selected for the derivatives in order to address the electron-donating/withdrawing effect on the strength of the molecule-metal interaction. The comparison of the cis and trans-derivatives revealed interactions of different nature between the molecules and the $\mathrm{Au}(111)$ surface. We have been able to disentangle these interactions analyzing the charge density difference plots, the projected density of states (pDOS) and the non-covalent interactions (NCI). For the non-substituted thiourea and all the studied derivatives, besides the strong S-Au bond, it has been observed an $\mathrm{Au} \cdot \mathrm{H}-\mathrm{N}$ interaction similar to a hydrogen bonding, which is caused by the charge transfer from the molecules to the $\mathrm{Au}(111)$ surface inducing a charge counterbalancing: $\mathrm{Au}^{\delta-} \mathrm{H}^{\delta+} \mathrm{N}^{\delta-}$. Furthermore, $\pi$-Au interactions between the functional group of the derivatives and the $\mathrm{Au}(111)$ surface, mainly observed for the cis-derivatives, were highlighted by the NCI plots. In some particular cases it was also possible to distinguish long range interactions of the type lone pair-Au. The present results provide insight into a new kind of organic molecule-metal surface interaction, opening the way for the synthesis of potential nanodevices with diverse applications such as sensing (biosensors or atmospheric sensors), optoelectronics, pollution control, or energy conversion.
\end{abstract}

\title{
Introduction
}

Adsorption of organo-sulfur compounds on gold surfaces have drawn a great interest in fields such as single-molecule junctions, self-assembly and electronic devices. ${ }^{1-4}$ The strong Au-S chemical affinity has been used to design different molecule-metal systems from the adsorption of organosulphur compounds on gold surfaces, specially the $\mathrm{Au}(111)$ surface. ${ }^{5-7}$ In particular, when this kind of compounds present $\pi$ delocalized electrons, they exhibit 
various interesting functionalities (e.g. photochromism, magnetism, etc.), which make them ideal active components to build up nanodevices. ${ }^{8-11}$ However, despite the strength of $\mathrm{Au}-\mathrm{S}$ bond, the geometry of molecules adsorbed on metal surfaces also depends on non-covalent interactions like the host-guest interactions, electrostatic interactions and metal-ligand interactions. ${ }^{12,13}$ The latter are closely related to the functionality of the molecular head group. Current experimental techniques like X-ray Photoelectron Spectroscopy (XPS) and Scanning Tunneling Microscopy (STM) can provide information about the geometry and the electronic structure of molecules adsorbed on metal surfaces. However, characterization of the molecule-metal interactions remains challenging. ${ }^{14,15}$ Thus, theoretical calculations must be used to investigate the nature of these interactions, and to shed light into the origin of the acting forces.

Thiourea derivatives are $\pi$ organo-sulfur molecules that arise as potential candidates to form stable molecule-metal hybrid inerfaces with the $\mathrm{Au}(111)$ surface showing interesting applications that are given by their catalytic, sensing, ionophore and luminescent properties. ${ }^{16-20}$ Furthermore, previous theoretical studies have demonstrated their strong interaction with other metal surfaces. ${ }^{16,20-22}$ The experimental reports related with the adsorption of these organo-sulfur molecules on gold surfaces are mainly focused in the single thiourea (TU). ${ }^{23-25}$ Theoretical aspects of the interaction between $\mathrm{TU}$ and $\mathrm{Au}(111)$ in an electrochemical context have been previously studied by Cheuquepán et al. using the cluster model approach. ${ }^{26}$ In this work thiourea/thioureate equilibrium is described, and supported by electrochemical experimental data, but the interactions between the TU and the $\mathrm{Au}(111)$ surface are not investigated. On the other hand, the theoretical reports regarding the adsorption of thiourea derivatives on $\mathrm{Au}(111)$ surface are scarce. ${ }^{27}$ Relevant aspects of the adsorption of $\mathrm{TU}$ and its derivatives on the $\mathrm{Au}(111)$ surface, like adsorption site, adsorption geometry and types of molecule-metal interactions remain undisclosed.

In the present work we examine the adsorption of TU and seven monosubstituted derivatives (see Fig. 1) on the $\mathrm{Au}(111)$ surface by means of first principle methods. The func- 
tional group connected to the thiourea core (NCSN) was selected in order to address the electrodonanting/withdrawing effect on the molecule-metal interactions. We also have considered the two most stable spatial configurations for each molecule, cis and trans, and the four possible configurations for the 2-furoyl derivative (FU). The goal of our work is to provide insight into the nature of the molecule-metal interactions of these systems and to establish a standard computational procedure for their characterization. We are also able to distinguish the effect of the functionalization of the thiourea-core over the stability. The use of charge difference plots, projected density of states (pDOS) and non-covalent interaction (NCI) analysis, allowed us to obtain a complete description of the interactions between the studied molecules and metal surface highlighting the bonding characteristics driving the formation of the strong metal-molecule anchorage. Overall, the NCI technique is essential to describe the long range interactions between molecules and surface. Nevertheless, despite the

well-known key role of dispersion forces in the stabilization of organic-metal interfaces, ${ }^{28,29}$ to our knowledge NCI mapping has not been previously applied to these type of systems. We finally show that the interplay between covalent and non-covalent interactions determine the adsorption of monosubstituted thiourea derivatives on the $\mathrm{Au}(111)$ surface.

\section{Computational Details}

All the calculations have been performed in the framework of Density Functional Theory (DFT) using the Gaussian09 $\operatorname{code}^{30}$ and Vienna Ab initio Simulation Package (VASP 5.4.4). ${ }^{31-34}$ Firstly we carried out a conformational analysis of the single thiourea and the derivatives at the gas phase level with Gaussian09 -revision E1- employing the M062X functional $^{35}$ and $6-31++\mathrm{G}(\mathrm{d}, \mathrm{p})$ basis set. In order to distinguish the unsubstituted and substituted nitrogen atoms in the derivatives we have labelled them as N1 and N2, respectively. The potential energy surfaces were obtained by constraining the rotation around the corresponding dihedral angles (N1-C-N2-H, H-N2- $\left.\mathrm{R}_{C}-\mathrm{X}\right)$ where $\mathrm{X}=\mathrm{R}_{C}$ or $\mathrm{R}_{O}$ and denotes 


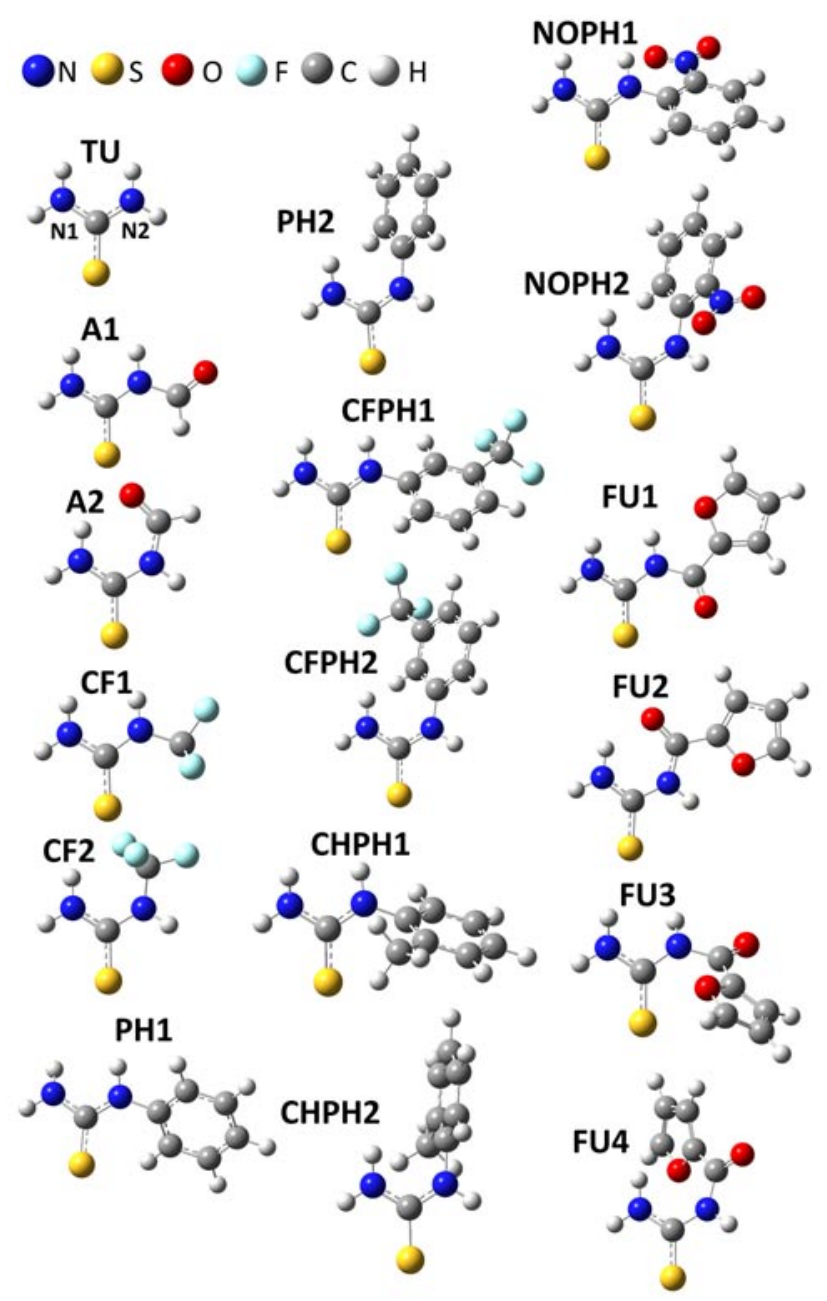

Figure 1: The thiourea molecule (TU) and the seven derivatives studied in the present work: formyl (A), trifluoromethyl $(\mathrm{CF}), o$-tolyl $(\mathrm{CHPH}), p$-trifluoromethylphenyl $(\mathrm{CFPH})$, $o$-nitrophenyl (NOPH), phenyl (PH) and 2-furoyl (FU). For the case of TU the notation of the unsubstituted (N1) and substituted (N2) nitrogens are depicted. The cis and trans configurations are denoted by odd and even numbers, respectively. Color balls have been used to represent the different atom types in the molecules. 
the $\mathrm{C}$ and $\mathrm{O}$ atoms in the functional group (see atom labels in Fig. 1). Under these constrains the remaining internal coordinates were fully optimized. Intervals of $30^{\circ}$ were used for the rotation of the dihedral angles in all the molecules. Frequencies were computed to characterize each stationary point of the conformational analysis as a minimum or a transition state. Then, we took the most stable geometries from the gas phase level (Fig. 1) as initial approach for the calculations under periodic boundary conditions (PBC) with VASP. The selected geometries were denoted with odd suffixes for cis configurations, and with even suffixes for trans configurations.

PBC calculations were performed under the generalized gradient approximation (GGA) ${ }^{36,37}$ using the functional of Perdew, Burke and Enzerhof (PBE). ${ }^{38,39}$ The ion-electron interactions were described through the projector augmented wave pseudopotential (PAW). ${ }^{40,41}$ The oneelectron Kohn-Sham wavefunctions were expanded in a plane-wave basis set with a $600 \mathrm{eV}$ cutoff energy. The $\mathrm{Au}(111)$ surface was modelled by a six-layer slab with a $(5 \times 3 \sqrt{3})$ and $(7 \times 3 \sqrt{3})$ supercells for the single thiourea and the derivatives, respectively. A vacuum of $\sim 20 \AA$ from the topmost layer along the $z$-axis was added to minimize interaction between periodic images. A larger periodicity of $14 \AA$ along the $x, y$ directions was included in order to neglect interactions between the molecules replica which allowed us to work in the single molecule regime. In addition, a dipole correction along the surface normal was included in all calculations for correcting the error introduced from the PBC. A $\Gamma$-point grid was used for the numerical integration in the reciprocal space of the supercells. Electron smearing was introduced following the Methfessel-Paxton technique ${ }^{42}$ at the first order level with a $\sigma=0.2 \mathrm{eV}$. All the energies reported here were extrapolated to $0 \mathrm{~K}$. For the calculation of the projected density of states (pDOS) a gaussian smearing with a sigma value of $0.1 \mathrm{eV}$ was used. Full relaxation of atomic positions in the three topmost layers of the slab and the molecules was allowed during geometry optimizations until reaching Hellmann-Feynman forces lower than $0.01 \mathrm{eV} / \AA$. The electronic iteration threshold was set to an energy of $10^{-5}$ $\mathrm{eV}$ for each ionic step. The adsorption energy $\left(E_{a d s}\right)$ for all the models has been calculated 
as:

$$
E_{a d s}=E_{m o l} / s l a b-\left(E_{s l a b}+E_{m o l}\right)
$$

where $E_{\text {mol/slab }}$ denotes the total electronic energy of the adsorbed molecule on the slab, $E_{\text {slab }}$ the energy of the clean slab and $E_{m o l}$ the energy of an isolated molecule.

The description of van der Waals interactions can not be neglected for the modeling of molecules adsorbed on metal surfaces. ${ }^{43}$ We have included the van der Waals forces with the density dependent dispersion correction (dDsC) scheme. ${ }^{44-46}$ Previous studies have proved the efficiency of the $\mathrm{dDsC}$ approach in the description of inter- and intramolecular forces. ${ }^{46,47}$ Furthermore, the dDsC scheme yields a more reliable lattice parameter for the gold surface. ${ }^{47}$ Thus, under the $\mathrm{dDsC}$ approach we attained, with a reasonable computational effort, an accurate modeling of molecule-metal interactions. ${ }^{48}$ Comparison of the PBE-dDsC scheme with the PBE-D3(BJ) ${ }^{49,50}$ is shown in the Supp.Info.

We have explored the presence of non-covalent interactions (NCI) ${ }^{51-53}$ between molecules and the metal surface, which are relevant in the adsorption process. For this purpose we have performed a topological analysis of the electron density using the CRITIC2 code. ${ }^{54,55}$ The inter- and intramolecular interactions have been discarded and only the molecule-metal interaction was considered to build up the NCI plots. Treating the molecules and the Au(111) surface as individual fragments allowed us to describe the NCI in the interface. The plots were obtained from the analysis of self-consistent electron densities. In order to identify the types of NCI, bonding or non-bonding, the sign of the second eigenvalue $\left(\lambda_{2}\right)$ of the electron-density Hessian matrix has been included in the representation. When $\lambda_{2}>0$ the NCI plots describes non-bonded interactions, while for $\lambda_{2}<0$ are bonded. ${ }^{51-53}$

Charge transfer process between the thiourea derivatives and the surface has been characterized using the Quantum Theory of Atoms in Molecules (QTAIM). ${ }^{56,57}$ In this model, based in the analysis of the electron density topology, real space is divided and assigned to the individual atoms in the system. Then, by integration of the density in each atomic volume, the atomic charge can be calculated. In this work, we have used the code developed 
by Henkelman and coworkers ${ }^{58-60}$ to carry out these calculations on the numeral grid of the electron density which is provided by VASP.

\section{Results and discussion}

The results of our theoretical study about the adsorption of monosubstituted thiourea derivatives on the $\mathrm{Au}(111)$ surface are presented as follows. First, we analyze the geometry and adsorption energy of the derivatives after the deposition on the $\mathrm{Au}(111)$ surface. Following this, we study the gain and depletion of electron density in the molecule-metal system through the charge difference plots for a visual inspection of the charge distribution. Quantification of the transferred charge as given with the QTAIM analysis is also presented. Further information concerning electronic structure and bonding is extracted from the Projected Density of States (pDOS) analysis. Finally, the Non-Covalent Interaction (NCI) plots are shown, allowing us to distinguish the presence of long range interactions between the thiourea derivatives and the $\mathrm{Au}(111)$ surface.

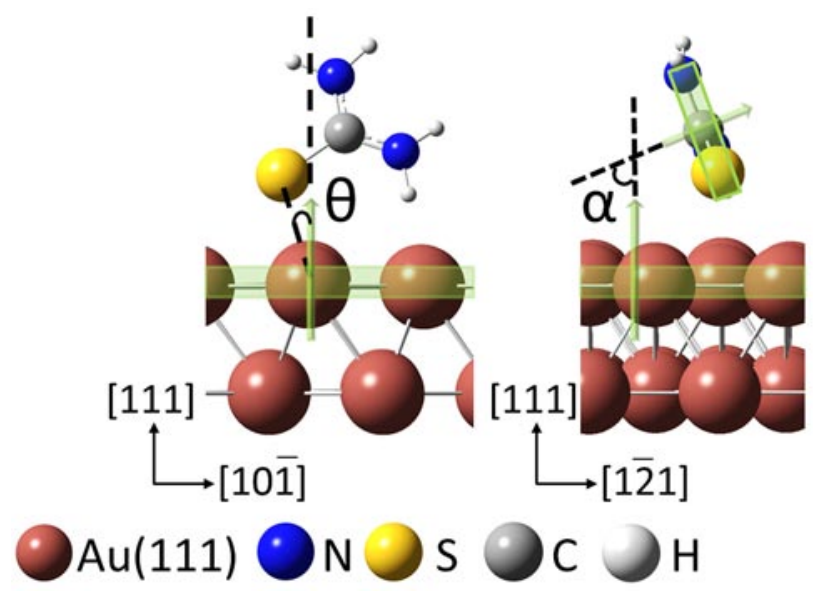

Figure 2: Representation of the tilt $(\theta)$, bending $(\alpha)$ angles and the planes formed by NCSN core and $\mathrm{Au}(111)$ outermost layer, both in green shaded. (Left) Schematic of the $\theta$ angle between the $\mathrm{Au}-\mathrm{S}$ distance and the normal to the $\mathrm{Au}(111)$ surface. (Right) Schematic of the $\alpha$ angle between the normal vectors of the NCSN plane and the $\mathrm{Au}(111)$ plane. In addition, the crystallographic direction are also included for a better visualization. 


\section{Adsorption Geometry and Energy}

The geometrical arrangement of $\mathrm{TU}$ and its derivatives with respect to gas phase upon adsorption on the $\mathrm{Au}(111)$ surface requires to consider other structural parameters, besides the bond distances. The study of the adsorption geometry of TU and the monosubstituted derivatives was carried out by defining the tilt $(\theta)$ and bending $(\alpha)$ angles, illustrated in Fig. 2. The $\theta$ angle (tilt) is defined as the angle formed between the Au-S bond and the surface normal; on the other hand, the $\alpha$ angle (bending) is described as the angle between the vector normal to the NCSN plane and the vector normal to the Au plane of the outermost layer. It is worth noting that $\theta$ varies from 0 to $90^{\circ}$, while $\alpha$ has a range from 90 to $-90^{\circ}$.

The optimized structure of the adsorbed TU, represented in Figure 2, shows that the plane formed by the NCSN core has $\theta=14.08^{\circ}$ and $\alpha=69.63^{\circ}$, being N1 closer to the surface than $\mathrm{N} 2$ in this case. In addition, the TU adsorption on the $\mathrm{Au}(111)$ surface occurs almost at the top site forming the S-Au bond. This TU adsorption geometry is in good agreement with previous theoretical reports at the DFT level ${ }^{26}$ and with the geometry proposed by García et al. from electrochemical measurements using gold electrodes. ${ }^{25}$ Furthermore, it resembles to the geometry observed in the formation of close-packed TU adlayers on $\mathrm{Au}(111) .{ }^{24}$

It has not been confirmed that the proximity of $\mathrm{TU}$ to the surface is due to a longrange interaction between N1 nitrogen and gold atoms on the surface. In order to probe the possible effect of the interaction between N1 and the metal surface in the TU geometry, we choose the thioformaldehyde molecule $\left(\mathrm{H}_{2} \mathrm{CS}\right)$. This molecule retained the $\mathrm{C}=\mathrm{S}$ bond character similarly to TU but it does not have nitrogen atoms in its structure. Values of $\theta=88.22^{\circ}$ and $\alpha=10.13^{\circ}$ obtained for $\mathrm{H}_{2} \mathrm{CS}$ on $\mathrm{Au}(111)$ surface, denote that the geometry is comparable to that obtained for TU (see Figure S3), thus indicating that $\mathrm{H}_{2} \mathrm{CS}$ and TU adsorption are of similar nature. Therefore, we can assume that TU and $\mathrm{H}_{2} \mathrm{CS}$ geometries are given by the interaction between the $\mathrm{HOMO}$, mainly located along the $\mathrm{C}=\mathrm{S}$ bond, and the extended states of the $\mathrm{Au}(111)$ surface (see Figure S2). Thus, confirming that the closeness of TU towards the surface is not by long range interaction between nitrogen N1 and gold 
atoms of the substrate. Further analysis on the electronic structure is presented in the next section to show the validity of this hypothesis.

The full optimized structures of the monosubstituted derivatives are presented in Figure 3, and Table 1 summarizes the computed $\theta$ and $\alpha$ parameters. Significant differences of the $\theta$ and $\alpha$ angles can be observed between the TU and the cis-monosubstituted derivatives. A close inspection to the values of $\theta$ and $\alpha$ illustrates a trend for the cis-derivatives, which allows us to sort the molecules in two groups. The first one is formed by A1, PH1, FU1 and FU3, and the obtained $\theta$ and $\alpha$ parameters reflect a lay-down arrangement respect to the $\mathrm{Au}(111)$ surface (see also Figure S4). In the second group, with CFPH1 and NOPH1, the molecules adopt a non lay-down arrangement with respect to the $\mathrm{Au}(111)$ surface. We can explain the different geometries of the cis-derivatives with a simple chemical approach. For CFPH1 and NOPH1, the presence of the $-\mathrm{CF}_{3}$ (trifluoromethyl) and $-\mathrm{NO}_{2}$ (nitro) functional group respectively, provokes a decrease in the electron density of the phenyl ring due to its electron-withdrawing effect. ${ }^{61,62}$ As a consequence, the interaction between the $\pi$ orbitals and the $\mathrm{Au}(111)$ surface is weaker in CFPH1 and NOPH1 than for the other cis-derivatives, thus leading them to adopt a non lay-down arrangement. Otherwise, CHPH1 presents a quasilay-down arrangement even though it has a $-\mathrm{CH}_{3}$ (methyl) functional group (see Figure $\mathrm{S} 4$ ). This behavior is likely caused by a steric effect between the sulfur atom of the NCSN core and $-\mathrm{CH}_{3}$ group on orto position. Finally, the $\theta$ and $\alpha$ angles computed for $\mathrm{CF} 1$ do not approach neither TU nor the rest of the cis-derivatives.

Table 1: Tilt $(\theta)$ and bending $(\alpha)$ angles, in degrees, for the single thiourea (TU) and the monosubstituted derivatives. The definition of $\theta$ and $\alpha$ is represented in Figure 2 .

\begin{tabular}{rrrrrrrrrr}
\hline \hline & TU & A1 & A2 & CF1 & CF2 & PH1 & PH2 & CFPH1 & CFPH2 \\
\hline$\theta$ & 14.08 & 37.30 & 18.95 & 34.81 & 38.36 & 13.52 & 8.07 & 22.86 & 17.32 \\
$\alpha$ & 69.63 & -31.21 & -78.73 & 54.11 & -34.38 & -17.30 & 64.16 & 48.32 & 56.71 \\
\hline \hline & CHPH1 & CHPH2 & NOPH1 & NOPH2 & FU1 & FU2 & FU3 & FU4 \\
\hline$\theta$ & 29.95 & 35.95 & 19.83 & 13.96 & 34.33 & 39.80 & 35.20 & 35.68 \\
$\alpha$ & 39.75 & 70.79 & 39.04 & 61.84 & 22.49 & 99.03 & -37.02 & 88.96 \\
\hline \hline
\end{tabular}



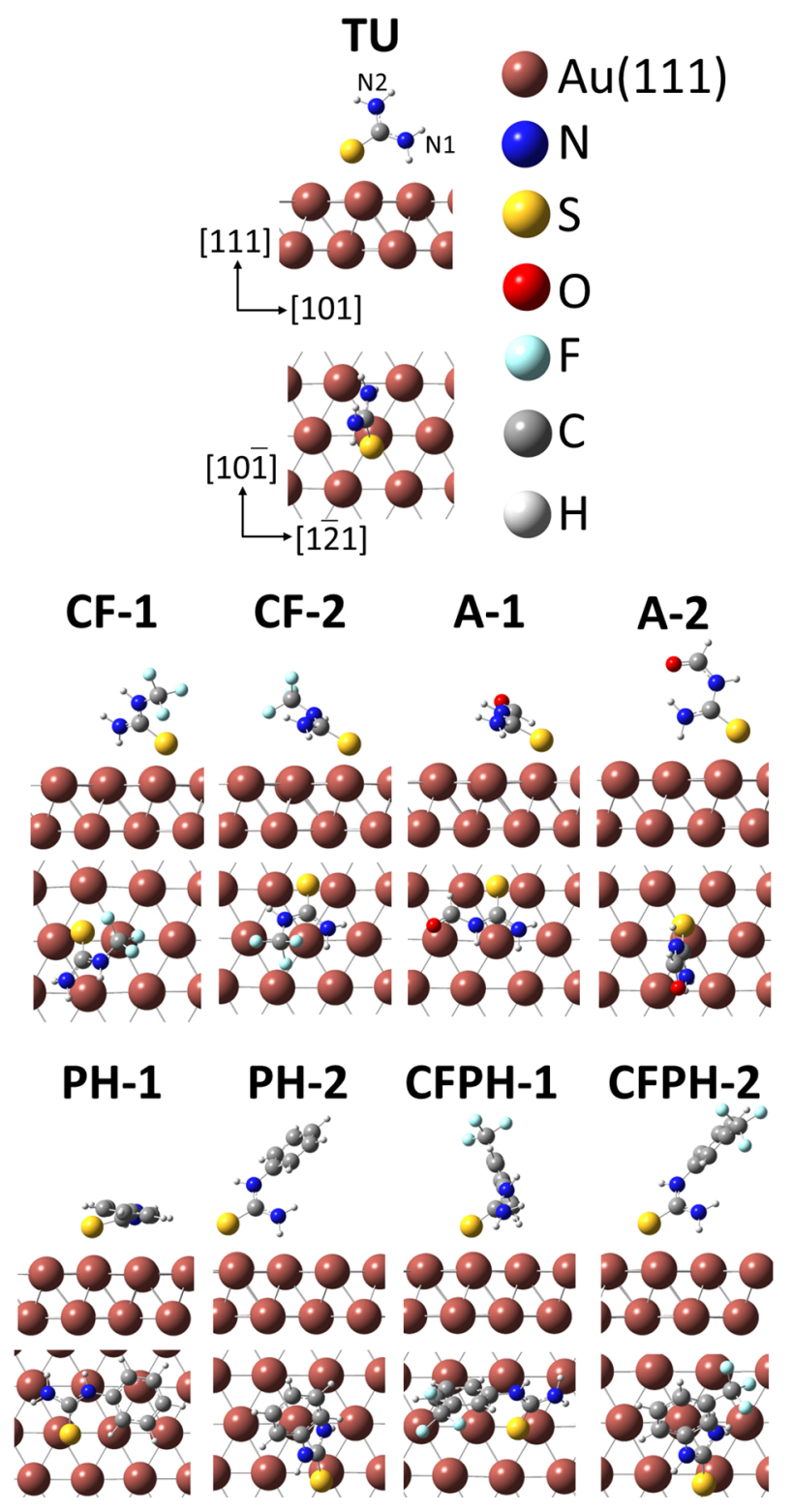

Figure 3: Side and top views of the optimized geometries of TU and A, CF, PH, CFPH derivatives. The labels N1 and N2 are illustrated in TU to distinguish the free and substituted nitrogen atom, respectively. 
For the trans-derivatives (even notation) the comparison of $\theta$ and $\alpha$ from Table 1 shows that these derivatives have a similar adsorption geometry to the one of TU (see also Figure S4), except for FU2, FU4 and CF2 derivatives. In the case of FU2 and FU4 derivatives the disagreement from the comparison of $\theta$ and $\alpha$ respect to TU is noticeable. We attribute this difference to the more complex structure of FU2 and FU4 in which intramolecular forces ${ }^{63,64}$ like hydrogen bonding (see Figure S4), can affect their interaction with the surface. Concerning CF2, the $\theta$ and $\alpha$ angles show to be closer to the cis-derivatives.

Figure 3 also shows the position of sulfur atom of the monosubstituted derivatives on the gold surface. One can appreciate that the sulfur atom is adsorbed close to the on-top site with a slight displacement around this position. By contrast, CF2 and A1 adsorption sites are close to bridge sites (see Figure S4). The change of adsorption site can be attributed to the electron-withdrawing effect of these substituents on the NCSN core and to their spatial configuration, with an almost lay-down geometry with respect to the surface (see Figure 3). The electron-withdrawing effect of the $-\mathrm{CF}_{3}$ (trifluoromethyl) and - $\mathrm{CHO}$ (formyl) groups decrease the electron density around the sulfur atom modifying the effective interaction between its HOMO orbital and the $\mathrm{Au}(111)$ surface (see Figure S1 and S2). On the other hand, the almost lay-down arrangement of the functional groups, $-\mathrm{CF}_{3}$ in $\mathrm{CF} 2$ and $-\mathrm{COH}$ in A1, can provoke the appearance of other molecule-metal interactions, which also affect the Au-S anchoring. Similar adsorption of the sulfur atom at the bridge site has been also observed for FU1 and FU3, in spite of having an electron-donating character. In this case, the position of the sulfur atom is, most likely, conditioned by the interaction between the carbonyl group $\mathrm{C}=\mathrm{O}$ and the surface. Consequently, it imposed an arrangement where the adsorption occurs close to bridge site (see Figure S4 and further discussion in the Supp.Info.).

From Table 2, it can be seen that the C-S bond distance inside the NCSN core is increased for all the derivatives and TU with respect to the gas phase structure, which indicates an enhancement of electron population in the LUMO orbitals of the molecules. In fact, the LUMO and LUMO +1 orbitals in the gas phase show an antibonding character along 
Table 2: Bond distances, in $\AA$, of the thiourea derivatives before and after the adsorption: C-S, C-N1, C-N2 and Au-S.

\begin{tabular}{llll|llll}
\hline \hline & \multicolumn{3}{c|}{ Gas phase } & \multicolumn{4}{c}{ Adsorption } \\
\hline & C-S & C-N1 & C-N2 & C-S & C-N1 & C-N2 & Au-S \\
TU & 1.661 & 1.368 & 1.368 & 1.703 & 1.335 & 1.354 & 2.462 \\
A1 & 1.653 & 1.358 & 1.392 & 1.708 & 1.339 & 1.367 & 2.592 \\
A2 & 1.655 & 1.343 & 1.400 & 1.692 & 1.323 & 1.393 & 2.471 \\
CF1 & 1.645 & 1.369 & 1.390 & 1.700 & 1.336 & 1.370 & 2.511 \\
CF2 & 1.654 & 1.352 & 1.394 & 1.712 & 1.331 & 1.365 & 2.538 \\
PH1 & 1.658 & 1.379 & 1.374 & 1.707 & 1.354 & 1.354 & 2.508 \\
PH2 & 1.665 & 1.361 & 1.375 & 1.709 & 1.332 & 1.359 & 2.464 \\
CFPH1 & 1.656 & 1.374 & 1.378 & 1.705 & 1.345 & 1.357 & 2.474 \\
CFPH2 & 1.669 & 1.351 & 1.372 & 1.706 & 1.334 & 1.361 & 2.471 \\
CHPH1 & 1.657 & 1.376 & 1.373 & 1.713 & 1.350 & 1.349 & 2.517 \\
CHPH2 & 1.665 & 1.362 & 1.371 & 1.723 & 1.328 & 1.356 & 2.480 \\
NOPH1 & 1.661 & 1.365 & 1.381 & 1.703 & 1.346 & 1.361 & 2.493 \\
NOPH2 & 1.656 & 1.364 & 1.384 & 1.699 & 1.334 & 1.369 & 2.471 \\
FU1 & 1.643 & 1.375 & 1.394 & 1.707 & 1.344 & 1.365 & 2.588 \\
FU2 & 1.664 & 1.336 & 1.395 & 1.713 & 1.331 & 1.368 & 2.462 \\
FU3 & 1.648 & 1.366 & 1.398 & 1.712 & 1.342 & 1.368 & 2.587 \\
FU4 & 1.658 & 1.350 & 1.394 & 1.714 & 1.326 & 1.374 & 2.456 \\
\hline \hline
\end{tabular}

the C-S bond, explaining the enlargement of the bond distance (see Figure S2). On the other hand, the decreasing of C-N2 distance can be explained through the increment of the bonding character of LUMO and $\mathrm{LUMO}+1$ around the $\mathrm{C}-\mathrm{N} 2$ bond, due to its larger electron population. Despite the decreasing of the C-N1 bond distance, the LUMO and LUMO +1 orbitals show an antibonding character. However, the behavior of the C-N1 bond distance was unexpected, thus further analysis is required.

The ranging Au-S bond distance from 2.456 to $2.592 \AA$ shows a good agreement with those reported for others organosulfur compounds adsorbed on the $\mathrm{Au}(111)$ surface. ${ }^{65-67}$ Table 2 shows that Au-S bond distance is larger in the cis-derivatives than in trans (even notation). The CF1 and CF2 derivatives show the opposite trend (Au-S:CF2 > Au-S:CF1), which is consistent with CF2 adsorption geometry resemblance to a cis-derivative.

Figure 4 shows the effect of cis-trans configurations over the Au-S bond distance considering the $\mathrm{Au}-\mathrm{N} 2$ height, i.e. the closeness of the functional groups to $\mathrm{Au}(111)$ surface. 

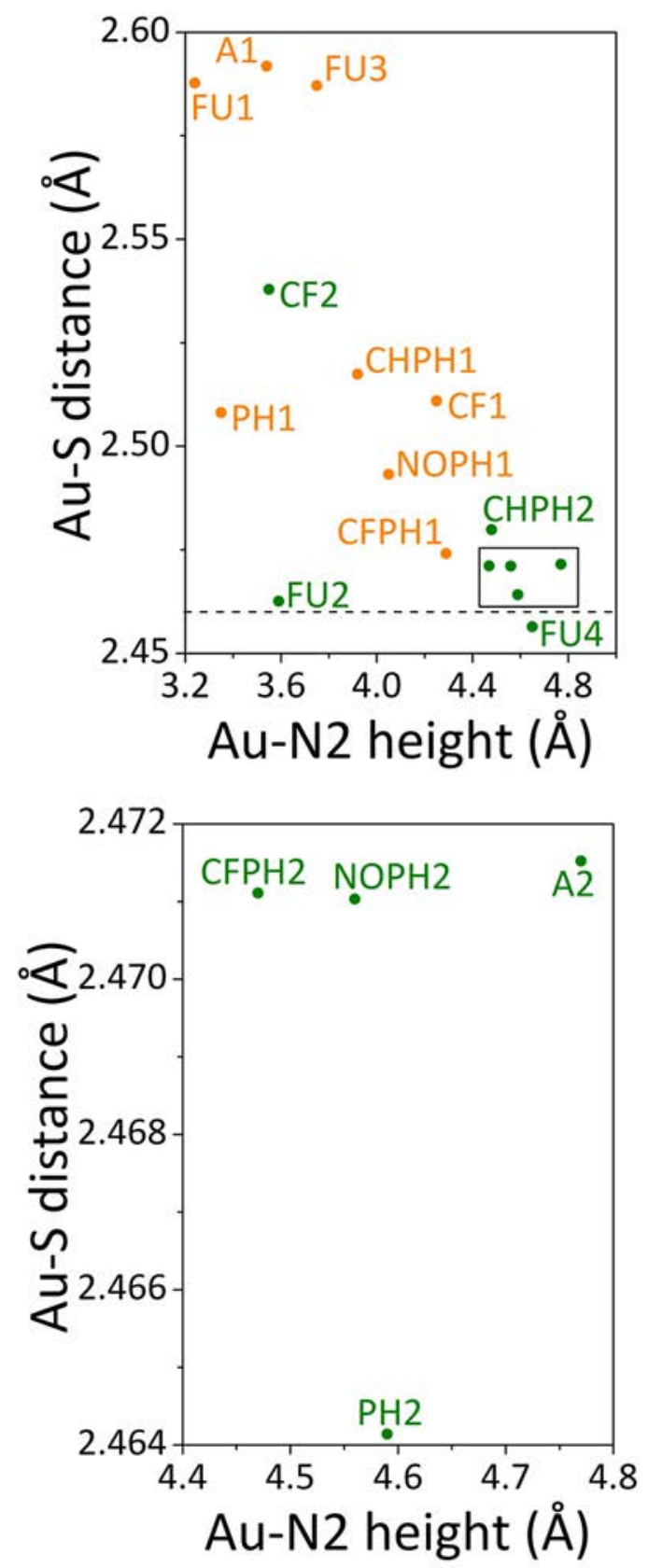

Figure 4: Correlation between the average height from the outermost layer of $\mathrm{Au}(111)$ surface to atom N2 (Au-N2 height) with the nearest distance Au-S. The cis and trans derivatives are represented by orange and green color, respectively. The horizontal dash line represents the distance Au-S of TU. Zooming of the data highlighted in the black square is represented in the bottom graphic. 
The Au-S bond distance of the TU is shown as a dashed line in the figure. The Au-S bond distances of the cis-derivatives increased with the approach of the functional group to the surface or the reduction of the Au-N2 height. The Au-N2 height of the trans-derivatives is above $4.4 \AA$ with Au-S bond distances similar to TU except for CF2 and FU2. CF2 has the lower value of Au-N2 height, below $3.6 \AA$ indicating a cis-like behaviour. The Au-N2 height of $\mathrm{CF} 2$ could be induced by a interaction between the $\pi-\mathrm{CF}_{3}$ orbitals and the $\mathrm{Au}(111)$ surface. ${ }^{68}$ On the other hand, 2-furoyl group gets closer to surface without affecting the Au-S bond distance due to the specific adsorption geometry of FU2 (see Table 2 and Figure S4). The Au-S bond distance is larger in cis-derivatives than trans-derivatives, except for CF1 and FU2 (see Table 2).

We have been able to evaluate the effect of Au-S distance and Au-N2 height on the adsorption energy, which consequently reflects the strength of the molecule-metal interaction. The adsorption energy has been calculated for each monosubstituted derivatives and it was correlated with the parameters $\mathrm{Au}-\mathrm{S}$ distance and $\mathrm{Au}-\mathrm{N} 2$ height. Figure 5 displays the $\mathrm{Au}-\mathrm{S}$ distance and $\mathrm{Au}-\mathrm{N} 2$ height as a function of the adsorption energy for each cis and trans-derivatives. There is not systematic trend in the adsorption energy respect to any of these distances, but it is possible to identify three regions. Figure 5a, first region, shows adsorption energy of the A1 and CF1 cis-derivatives $<0.05 \mathrm{eV}$. Being A1 more stable than $\mathrm{CF} 1$, which is attributed to the closeness of its functional group - $\mathrm{COH}$ to the surface (see Au-N2 height). Although the difference in adsorption energy between the A2 and CF2 transderivatives is only of $0.05 \mathrm{eV}, \mathrm{CF} 2$ presents higher stability from the effect of the closeness of $-\mathrm{CF}_{3}$ group to the surface. However, $\mathrm{A}$ and $\mathrm{CF}$ derivatives exhibit similar adsorption energies regardless of the cis-trans configuration, which highlights the predominant effect of the electron-withdrawing nature of the - $\mathrm{CHO}$ (formyl) ${ }^{69,70}$ and $-\mathrm{CF}_{3}$ (trifluoromethyl) ${ }^{71,72}$ functional groups on the NCSN core. Finally, Figure 5a also shows the presence of the FU4 derivative energetically close to A2, which is due to the fact that FU4 and A2 present similar Au-N2 heights. 


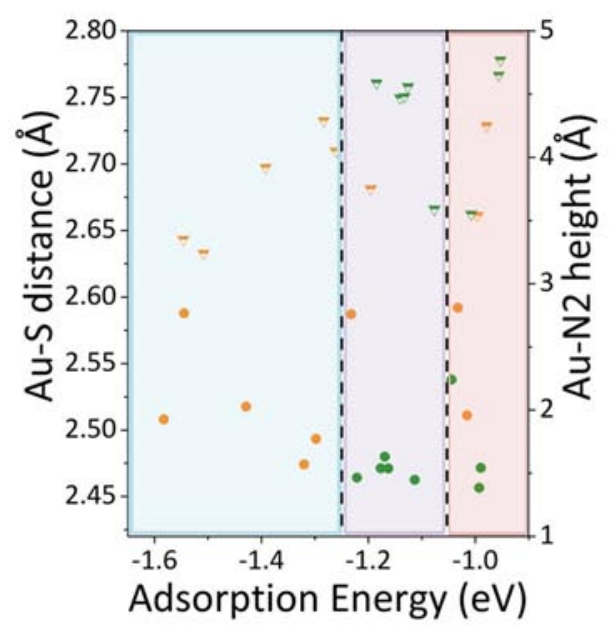

c)

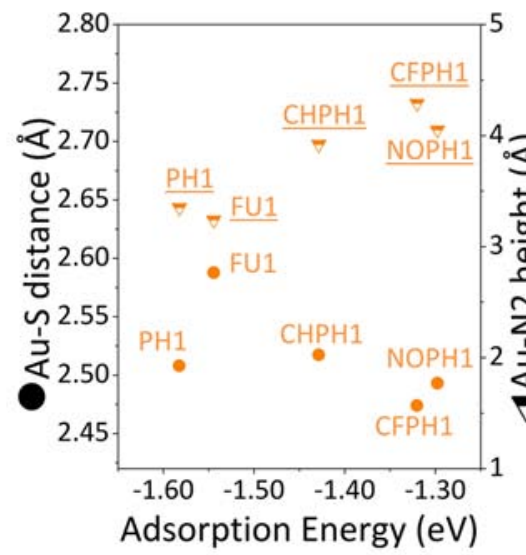

b) $\mathrm{TU}=-1.096 \mathrm{eV}$

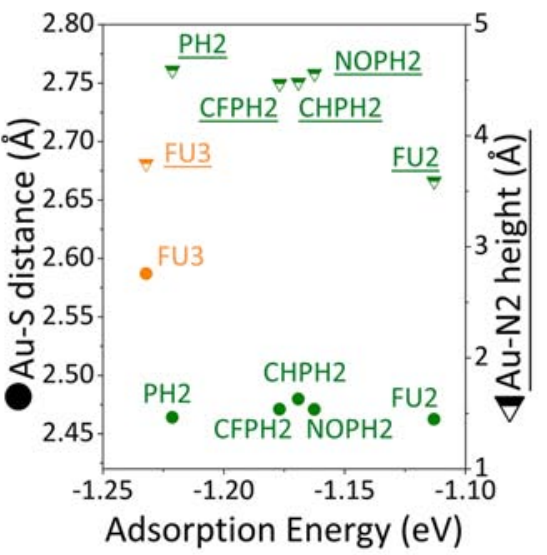

a)

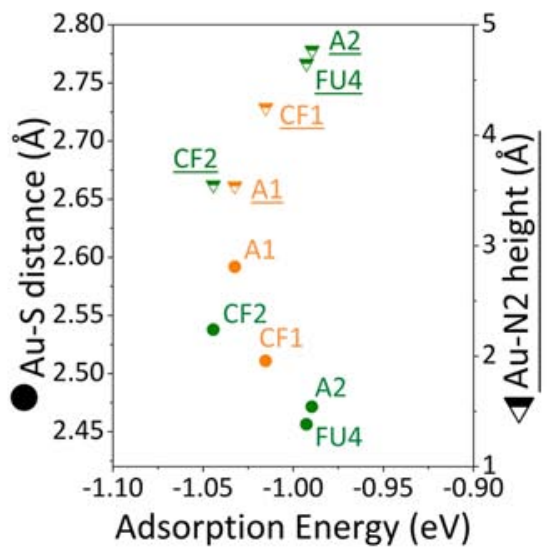

Figure 5: Adsorption energy of the monosubstituted derivatives correlated with the Au-S distance and the Au-N2 height. (Top) Representation in colors of the three regions of the adsorption energy under study. (Bottom) Zoom in the three regions of adsorption energy: (a) -0.90 to $-1.10 \mathrm{eV}$, (b) -1.10 to $-1.25 \mathrm{eV}$, (c) -1.25 to $-1.65 \mathrm{eV}$. The $\mathrm{Au}-\mathrm{N} 2$ height have been underlined for a better visualization. Orange and green color corresponds to the cis and trans-derivatives, respectively. The adsorption energy of TU have been inserted for a better comparison. 
The second region, shown in Figure 5b, exhibits mainly trans-derivatives with lower adsorption energies than TU, specially those with a phenyl ring on their structure. The adsorption energy difference between the phenyl trans-derivatives NOPH2, CHPH2 and CFPH2 is of $\sim 0.05 \mathrm{eV}$. However, the PH2 derivative is slightly more stable than the other phenyl trans-derivatives. They present almost the same values of Au-N2 height and Au-S distance. Thus, we can consider that the stability order observed for these derivatives is caused by two factors: (i) the electron-donating/withdrawing effect of the functional groups connected to the phenyl ring and (ii) the relative orientation of the phenyl ring with respect to the surface (see Figures S1, S4 and Table 1). According to Figure S4, the orientation of the phenyl ring is similar for PH2, CFPH2 and NOPH2, but the electron-withdrawing effect of $-\mathrm{CF}_{3}$ and $-\mathrm{NO}_{2}$ functional groups ${ }^{61,62}$ make CFPH2 and NOPH2 less stable than PH2. The phenyl orientation of CHPH2 strongly differs from that in PH2, reducing its stability, but the electron-donating effect of the $-\mathrm{CH}_{3}{ }^{73}$ functional group probably causes that it remained energetically close to CFPH2 and NOPH2. For these trans-derivatives such trend implies that molecule-metal interactions can be affected by the modification of the $\pi$ electron density of the phenyl ring and its orientation with respect to the surface. Another trans-derivative observed in Figure 5b is FU2 that appears at higher adsorption energy than the phenyl trans-derivatives. FU2 is less stable than the phenyl trans-derivatives, despite showing the 2-furoyl group at a smaller Au-N2 height, due to its lower electron-donating effect over the NCSN core and its non lay-down arrangement (see Figure S1 and S4).

In the particular case of FU3, an almost similar adsorption energy that PH2 (-1.23 vs $-1.22 \mathrm{eV}$ ) is observed as consequence of the $\mathrm{S}$. . O repulsive interaction, which breakdowns the lay-down arrangement of the molecule reducing the $\pi$-Au(111) interaction. The FU3 behavior is unexpected, it should have a lower adsorption energy because it is a cis-derivative with the functional group closer to the surface. The breakdown was distinguished with the comparison of the FU1 and FU3 dihedral angle $\left(\mathrm{R}_{O}-\mathrm{R}_{C^{-}}-\mathrm{R}_{C}-\mathrm{R}_{O}\right)$ where $\mathrm{R}_{C}$ and $\mathrm{R}_{O}$ denoted the $\mathrm{C}$ and $\mathrm{O}$ atoms of the 2-furoyl group. The dihedral angle showed values of $180^{\circ}$ and $158^{\circ}$ 
for FU1 and FU3, revealing the disruption of the lay-down arrangement in FU3.

The rest of cis-derivatives appear in Figure 5c. The decreasing of adsorption energy is caused by the increment of the electron-donating effect of the functional group and an enhancement of the $\pi$-Au(111) interaction. The latter is due to the coupling between the $\pi$ molecular orbitals and the $\mathrm{Au}(111)$ surface, which can be more effective in those derivatives with a marked lay-down arrangement. Consequently, FU1 and PH1 were found as the most stable derivatives, both have the strongest electron-donating effect on the NCSN core and with a lay-down arrangement close to $180^{\circ}$ or $0^{0}$ respect to the $\mathrm{Au}(111)$ surface (see Figure $\mathrm{S} 4$ and $\alpha$ in Table 1). Interestingly, PH1 and FU1 exhibits Au-S distance higher than TU $(2.45 \AA)$ which means that the enhancement of the molecule-metal interaction allows the anchoring of sulfur at larger distances without implying a loss of stability.

In summary, the cis-derivatives predominantly adopted a lay-down arrangement with the exception of CFPH1 and NOPH1 derivatives. The lay-down arrangement increased the stability of the cis-derivatives adsorbed on the $\mathrm{Au}(111)$ surface due to the enhancement of the molecule-metal interaction caused by the coupling between $\pi$ orbitals and the $d$ extended metal states. Nevertheless, we have proved that the stability of molecule-metal system in the cis-derivatives not only depends of the $\pi$ - $d$ coupling but also on the electrondonating/withdrawing nature of the functional group connected to the NCSN core. The electron-withdrawing groups trifluoromethyl $\left(-\mathrm{CF}_{3}\right)$ and formyl $(-\mathrm{COH})$ can modify the electron density inside the NCSN core, specially on the sulfur, weakening the molecule-metal interaction. On the other hand, the presence of an electron-donating group increments the electron density inside the NCSN core, which leads to a strengthening of the molecule-metal interaction.

On the contrary, the trans-derivatives adopted a non lay-down arrangement with the functional groups far from the $\mathrm{Au}(111)$ surface. The non lay-down arrangement made less stable the trans-derivatives but with $\mathrm{Au}-\mathrm{S}$ bond distances smaller, which is attributed to the weakening of $\pi$ - $d$ interaction and the predominance of Au-S interaction. The effect of 
electron-donating groups retains the enhancement of the electron density inside the NCSN core while electron-withdrawing groups lead to the opposite trend. The molecule-metal interaction of the phenyl trans-derivatives also depends on the orientation of the phenyl ring.

In a simple picture, the closeness of the functional groups to the $\mathrm{Au}(111)$ surface and their electron-donating effect enhance the adsorption process, thus, strengthening the moleculemetal interaction. These two effects are separately analyzed in the next sections. We first focus on the charge transfer effects and their consequences in the electronic structure changes, and then we elucidate the weak interactions between the functional groups and the surface through a NCI analysis.

\section{Charge Transfer}

The S-Au interaction plays an important role in the stabilization of the molecule-metal system. The high chemical affinity shown by the Au-S bond is characterized by the deformation of the large electronic valence shell of the sulfur atom towards the surface. ${ }^{5,74,75}$ This effect can be evaluated with charge density difference plots $(\Delta \rho)$. Figure 6 shows the induced electron density redistribution, gain and depletion of electrons, induced by the adsorption of the molecule on the surface. $\Delta \rho$ also reflects all the other electronic interactions, besides Au$\mathrm{S}$, that occur between the molecules and the $\mathrm{Au}(111)$ surface. An electron density gaining (green) can be observed on the molecules, mainly in the NCSN core, which is the region that corresponds to the LUMO and LUMO +1 orbitals of the molecules in the gas phase level (see Figure S2 and S5). Taking into account the symmetry of LUMO and LUMO +1 orbitals of the molecules, we can assume that the molecule-metal interaction leads to a charge transfer from the $\mathrm{Au}(111)$ surface to these molecular orbitals. The charge transfer towards the molecules causes the change in C-S and C-N2 bond distances after the adsorption (see Table 2 and the previous related discussion). Therefore, the close relation between the changes in the adsorption geometries and the LUMO and LUMO +1 symmetry points out the charge transfer 
process as a key ingredient to understand the nature of the molecule-metal interaction.

Another common aspect in the $\Delta \rho$ plots of Figure 6 is the appearance of gain and depletion of electron density in the regions highlighted with a black arrow. These regions correspond to lateral interactions $\mathrm{Au} \cdot \cdots \mathrm{H}-\mathrm{N} 1$ caused by the charge donation from the molecule to the surface, which generate a $\mathrm{Au}^{\delta-} \mathrm{H}^{\delta+} \mathrm{N} 1^{\delta-}$ counterbalancing with similar characteristics to a hydrogen bond. In addition, the Au-hydrogen interaction modifies the antibonding character of the LUMO orbitals along the C-N1 bond, which explains the decreasing of this bond distance as observed in Table 2. The interaction of the FU2 derivative takes place through two hydrogen atoms: H-N2 and one of the hydrogen atoms of the 2-furoyl group (see the double arrow in Figure 6 pointing each interaction). Therefore, in this case charge donation does not occur through the H-N1 bond. Consequently, the antibonding character of LUMO along the $\mathrm{H}-\mathrm{N} 1$ bond in FU2 almost remains unperturbed after the adsorption. Such charge donation process causes a negligible decrease $(\sim-0.005 \AA)$ of the bond distance (see Table 2).

The effect of the cis-trans derivatives configurations can be mainly highlighted in the changes of the $\Delta \rho$ plots observed in the functional group. In general, for the trans derivatives, small modifications in $\Delta \rho$ around the functional group are appreciated, which illustrates the lack of interaction with the $\mathrm{Au}(111)$ surface (see Figure S5). This effect differs for CF2, where a strong electron density depletion around the functional group denotes the interaction between the trifluoromethyl group $\left(\mathrm{CF}_{3}\right)$ and the $\mathrm{Au}(111)$ surface. On the other hand, all cis-derivatives exhibit a much larger enhancement in the gain and depletion of the electron density around the functional group in comparison with the trans-derivatives. Depletion for A1, PH1, CHPH1, FU1 and FU3 occurs in the plane containing the $\pi$ electrons of the molecules; we attribute this behavior to an electron donation from the $\pi$ orbitals of the molecules to the surface. On the contrary, electron density depletion in CFPH1 and NOPH1 takes place in the hydrogen atoms of the phenyl ring, which describes a weaker electron donation process with respect to other cis-derivatives. 


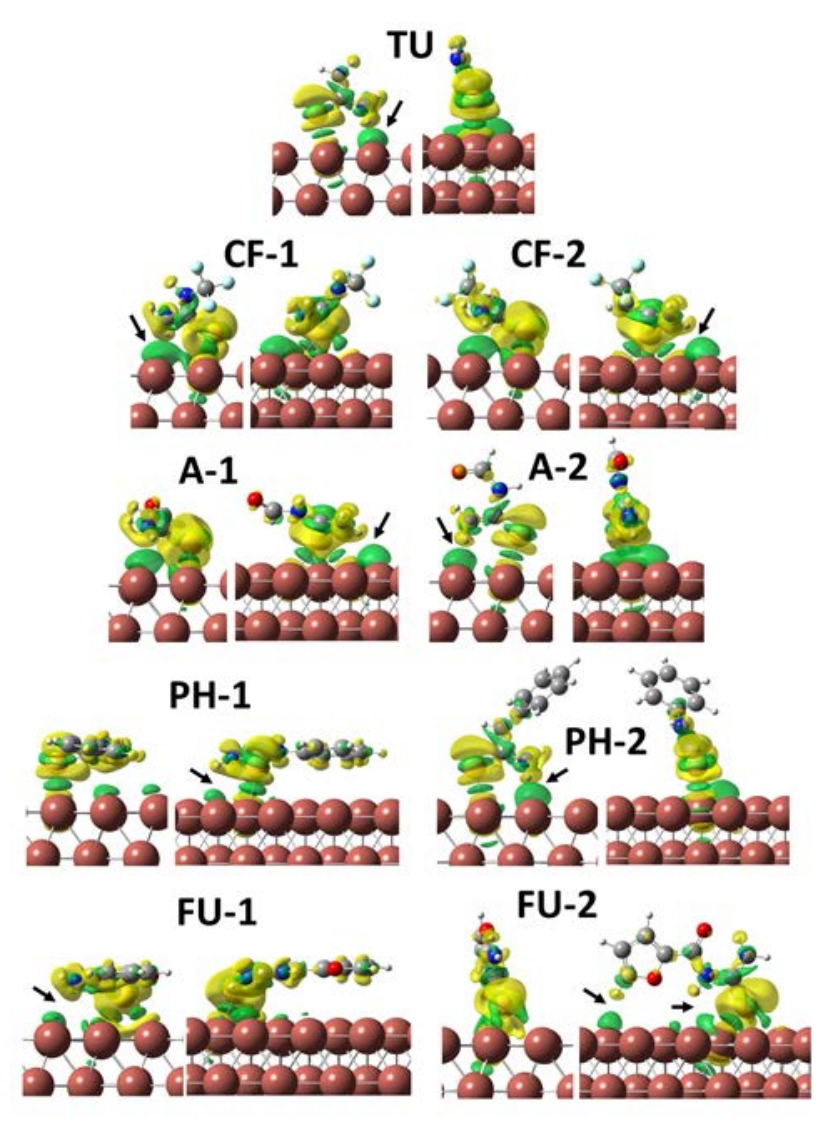

Figure 6: Electron density difference plots of TU and CF, A, PH, FU1, FU2 derivatives along the [101] (right) and [12̄1] (left) directions. Yellow regions stand for the depletion of electrons and green for the gaining. Cutoff isovalue $=0.01 e^{-} / \mathrm{Bohr}^{3}$. The black arrow depicts the regions where a Au-.H-N1 interaction appears. For further information see Figure S5. 
Charge transfer between the molecule and the surface has been quantified using the Quantum Theory of Atoms in Molecules. ${ }^{56}$ Table 3 summarizes the calculated values of the transferred charge from the molecule towards the surface $(\Delta q)$ after the adsorption, and the variation of the charge in the atoms of the NCSN core and in the functional group $(R)$ with respect to the molecule in the gas phase. Herein, it is observed loss of electron density in all the molecules after the adsorption, which generates a negatively charged $\mathrm{Au}(111)$ surface, mainly localized at the interface. As can be seen in Table 3, the $\mathrm{C}$ atom in the thiourea core displays a noticeable loss of electron density in comparison with the $\mathrm{S}$ and $\mathrm{N}$ atoms, after the adsorption. Similarly, the functional group $(R)$ connected to the NCSN core mainly exhibits a decreasing of charge after the adsorption, except for the FU2 derivative where a slight increment of charge in $R$ is observed.

Table 3: Charge transfer from the surface to the molecules $(\Delta q)$, charge allocated in the S, C, N1, N2 atoms and also for the functional group R connected to the thiourea core. All the units are in $e^{-}$.

\begin{tabular}{rr|r|r|r|r|r}
\hline \hline TU & -0.317 & -0.009 & -0.347 & -0.011 & -0.052 & \\
A1 & -0.271 & -0.026 & -0.198 & 0.003 & 0.008 & -0.037 \\
A2 & -0.258 & -0.030 & -0.213 & 0.006 & 0.048 & -0.064 \\
CF1 & -0.273 & -0.028 & -0.208 & -0.005 & 0.006 & -0.028 \\
CF2 & -0.305 & -0.038 & -0.263 & -0.012 & -0.005 & -0.009 \\
PH1 & -0.325 & 0.001 & -0.260 & -0.013 & 0.046 & -0.082 \\
PH2 & -0.323 & -0.090 & -0.174 & 0.015 & -0.039 & -0.025 \\
CFPH1 & -0.304 & -0.038 & -0.212 & -0.027 & 0.046 & -0.057 \\
CFPH2 & -0.310 & -0.082 & -0.184 & -0.018 & 0.035 & -0.052 \\
CHPH1 & -0.331 & -0.035 & -0.216 & -0.041 & -0.054 & -0.048 \\
CHPH2 & -0.355 & -0.070 & -0.225 & -0.024 & -0.043 & -0.040 \\
NOPH1 & -0.279 & -0.061 & -0.218 & 0.003 & 0.019 & -0.035 \\
NOPH2 & -0.279 & -0.068 & -0.182 & 0.030 & -0.036 & -0.042 \\
FU1 & -0.314 & 0.061 & -0.255 & -0.021 & -0.038 & -0.045 \\
FU2 & -0.293 & -0.057 & -0.275 & -0.024 & 0.045 & 0.003 \\
FU3 & -0.281 & 0.002 & -0.235 & -0.036 & -0.040 & -0.026 \\
FU4 & -0.291 & -0.070 & -0.251 & 0.019 & -0.017 & -0.032 \\
\hline \hline
\end{tabular}

All these features lead us to conclude that two charge transfer processes occur after the adsorption: (i) electron donation from the molecule towards the surface (stronger) and (ii) 
electron back-donation from the surface to the molecule (weaker). More specifically, electron transfer corresponds to donation from the NCSN core to the $\mathrm{Au}(111)$ surface (HOMO $\rightarrow \mathrm{Au})$. The TU and CF derivatives show lower $\pi$ conjugation ${ }^{76,77}$ than the rest of the molecules, but we can observe charge transfer comparable to the observed for CFPH and NOPH derivatives. This behavior is due to the predominant electron donation process through $\mathrm{HOMO} \rightarrow \mathrm{Au}$. Analyzing the energies of the HOMO of each thiourea derivatives in the gas phase, we have found a direct correlation with the transferred charge. Notice in Figure 7 an almost linear dependence between the HOMO energy in gas phase and $\Delta q$. The charge transfer from the molecules to the $\mathrm{Au}(111)$ surface increases with the shifting towards higher energies of the HOMO orbital. The PH and CHPH derivatives with stronger electron donating character transfer more charge than NOPH derivatives. This implies that the $\mathrm{HOMO} \rightarrow \mathrm{Au}$ electron donation depends on the electronic density around the sulfur atom, which is controlled by the effect of electron donating/withdrawing character of the functional groups. Two particular cases, CF2 and FU1, highlighted in Figure 7, deviate from the HOMO- $\Delta q$ linear trend. In the case of FU1 $\Delta q$ does not depend only on the HOMO orbital of the molecule, which is mainly located around the sulfur atom (see HOMO of FU1 in Figure S2), but also on the closeness of $\mathrm{C}=\mathrm{O}$ to the surface, since the electrodonating character of the 2-furoyl group causes an increase of the electron population around the carboxylic oxygen atom. On the other hand, for CF2, its almost lay-down arrangement enhances the $\pi$ - $d$ interaction between the $-\mathrm{CF}_{3}$ group and the $\mathrm{Au}(111)$ surface. Therefore, the electron donation occurs through two channels: HOMO $\rightarrow$ Au donation and $\pi \rightarrow$ Au donation. In summary, the total charge transfer process can be interpreted as a double $\pi \rightarrow \mathrm{Au}$ and $\mathrm{HOMO} \rightarrow \mathrm{Au}$ donation accompanied by a $\mathrm{Au} \rightarrow \pi^{*}$ back-donation, whose strength will be dictated by the adsorption geometry of these molecules. Similar charge transfer process has been observed for various molecule-metal systems such as simple molecules like $\mathrm{CO}^{78,79}$ and $\mathrm{NO}^{80,81}$ as well as in more complex organic molecules. ${ }^{82,83}$

Another relevant property related to the charge transfer of the molecule-metal system is 


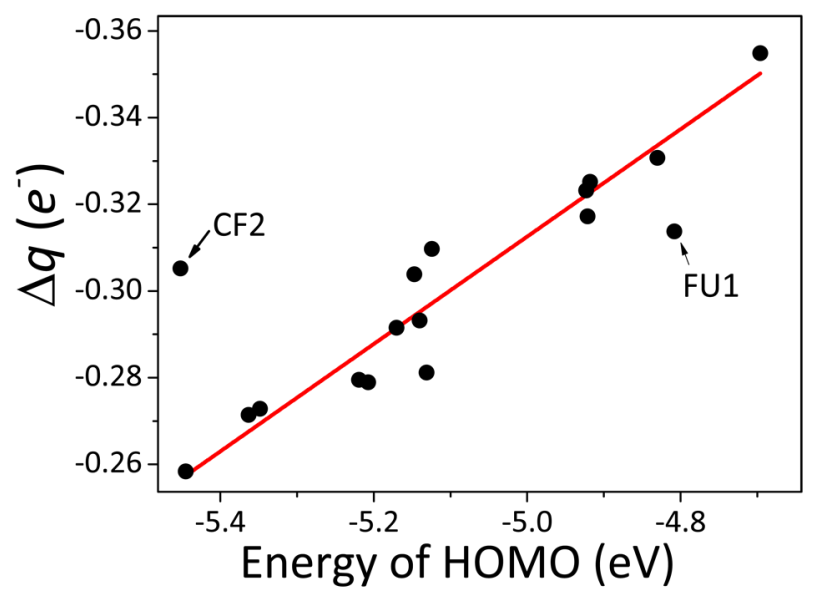

Figure 7: Correlation between the shifting of gas phase HOMO energy by the functional groups and the charge transfer towards the molecule $(\Delta q)$. The red line is used as guideline. The particular case of CF2 and FU1 are highlighted on the graphic. For more detailed information see Table S1.

the variation in the work function from the clean to the functionalized surface. The work function is sensitive to the charge reorganization produced in the interface, induced by the charge transfer process, and also to the modifications of the surface chemistry generated by the adsorbate. ${ }^{84-87}$ We have computed the work function variation of the $\mathrm{Au}(111)$ surface upon the adsorption $(\Delta \phi)$ using the standard procedure described by the equations:

$$
\begin{gathered}
\phi_{i}=E_{\text {vacuum }}-E_{F} \\
\Delta \phi=\phi_{f}-\phi_{0}
\end{gathered}
$$

where $E_{\text {vacuum }}$ is the Coulomb potential seen by the electrons in the vacuum of the slab, $E_{F}$ the Fermi energy; $\phi_{i}$ describes the general equation to determine the work function of the clean $\left(\phi_{0}\right)$ and modified $\left(\phi_{f}\right)$ surfaces, respectively. The results of $\Delta \phi$ are presented in Table 4.

The adsorption of thioureas derivatives on the $\mathrm{Au}(111)$ surface increases the work function, which points in the opposite direction as expected if one considers the observed charge transfer from the molecule to the surface. In principle, the charge transfer should generate a 

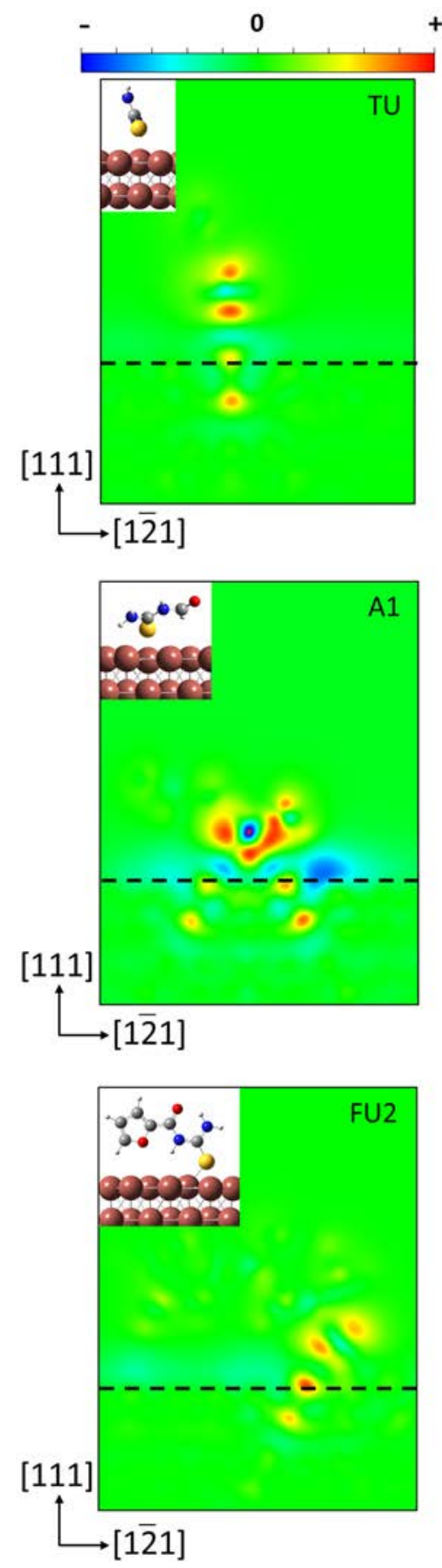

Figure 8: Cross section of the charge difference plots along the [111] direction for TU, A1 and FU2. Red (blue) regions represent the depletion (accumulation) of charge. The black dash line depicts the position of gold atoms in the topmost layer of the $\mathrm{Au}(111)$ surface. Insets are included to illustrate the schematic of the orientation selected to obtain the cross sections. 
Table 4: Comparison between the charge transfer from the molecules to the $\operatorname{Au}(111)$ surface $(\Delta q)$ and the variation of the work function $(\Delta \phi)$.

\begin{tabular}{ll|l}
\hline \hline & $\Delta q\left(e^{-}\right)$ & $\Delta \phi(\mathrm{eV})$ \\
\hline TU & -0.317 & 0.045 \\
A1 & -0.271 & 0.060 \\
A2 & -0.258 & 0.026 \\
CF1 & -0.273 & 0.067 \\
CF2 & -0.305 & 0.065 \\
PH1 & -0.325 & 0.062 \\
PH2 & -0.323 & 0.080 \\
CFPH1 & -0.304 & 0.064 \\
CFPH2 & -0.310 & 0.065 \\
CHPH1 & -0.331 & 0.092 \\
CHPH2 & -0.355 & 0.073 \\
NOPH1 & -0.279 & 0.064 \\
NOPH2 & -0.279 & 0.064 \\
FU1 & -0.314 & 0.062 \\
FU2 & -0.293 & 0.090 \\
FU3 & -0.281 & 0.066 \\
FU4 & -0.291 & 0.068 \\
\hline \hline
\end{tabular}

decrease of the work function due to the total positive and negative charges located outside and inside the $\mathrm{Au}(111)$ surface respectively. ${ }^{88}$ However, changes of the work function depend rather on the charge distribution in the interface, i.e. change of density dipole, than on the charge transfer process. ${ }^{86,89}$ As example, Figure 8 illustrates the electronic distribution in the interface of TU, A1 and FU2, observed in the charge difference plots of the plane along the [111] direction (see Figure S8 for all depictions). Note that the accumulation of charge does not occur only on the $\mathrm{Au}(111)$ surface, but also inside the molecule one can appreciate regions with negative charge far from the surface. As consequence, the charge redistribution produces an effective density dipole pointing inward the surface, which reinforces the work function of the metal surface. ${ }^{86,88}$

The charge distribution along the [111] direction in Figure 9 exhibits the presence of negatively charged regions far from the $\mathrm{Au}(111)$ surface. We have not considered the effect of the adsorbate-substrate distance on the work function change, as suggested in previous reports, ${ }^{90,91}$ taking into account that the negative regions in A1, FU2 and TU were almost 


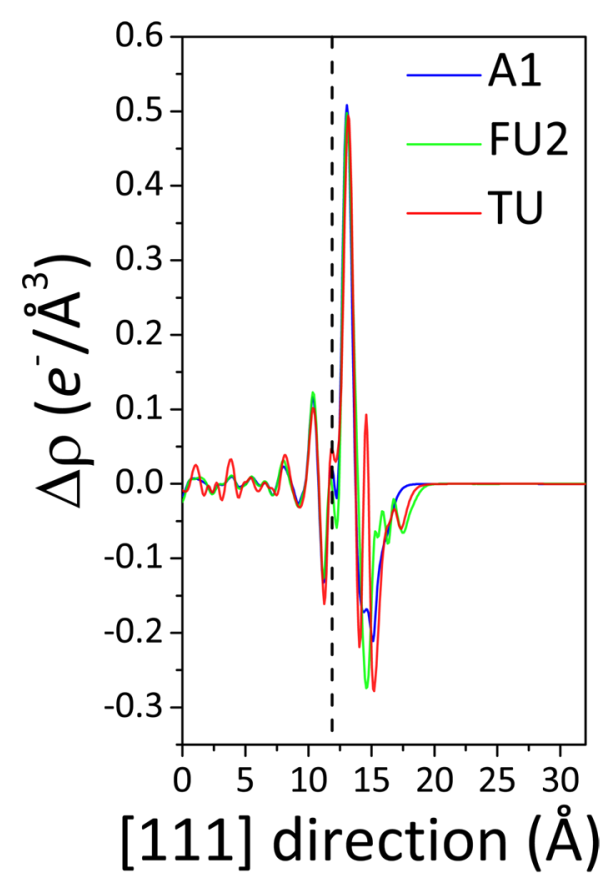

Figure 9: Charge density difference plotting along the [111] direction using the cross sections of A1, FU2 and TU in Figure 8. The vertical dash line indicates the approximate position of the topmost $\mathrm{Au}(111)$ layer. The charge density difference $(\Delta \rho)$ has been converted to $e^{-} / \AA^{3}$ for a better correlation with the displacement in the [111] direction. 
at $4 \AA$ from the surface. The integration of these maximum negative regions gave values of $-0.30 e,-0.27 e$ and $-0.18 e$ for $\mathrm{FU} 2, \mathrm{TU}$ and $\mathrm{A} 1$, respectively. The presence of a larger negative charge, caused by the increment of the density dipole, provokes the increasing of the metal work function, which explains the order of $\Delta \phi$ observed in Table 4 (FU2 > TU > A1). This analysis also explains the behavior observed in the rest of derivatives (see details in the Supp.Info.).
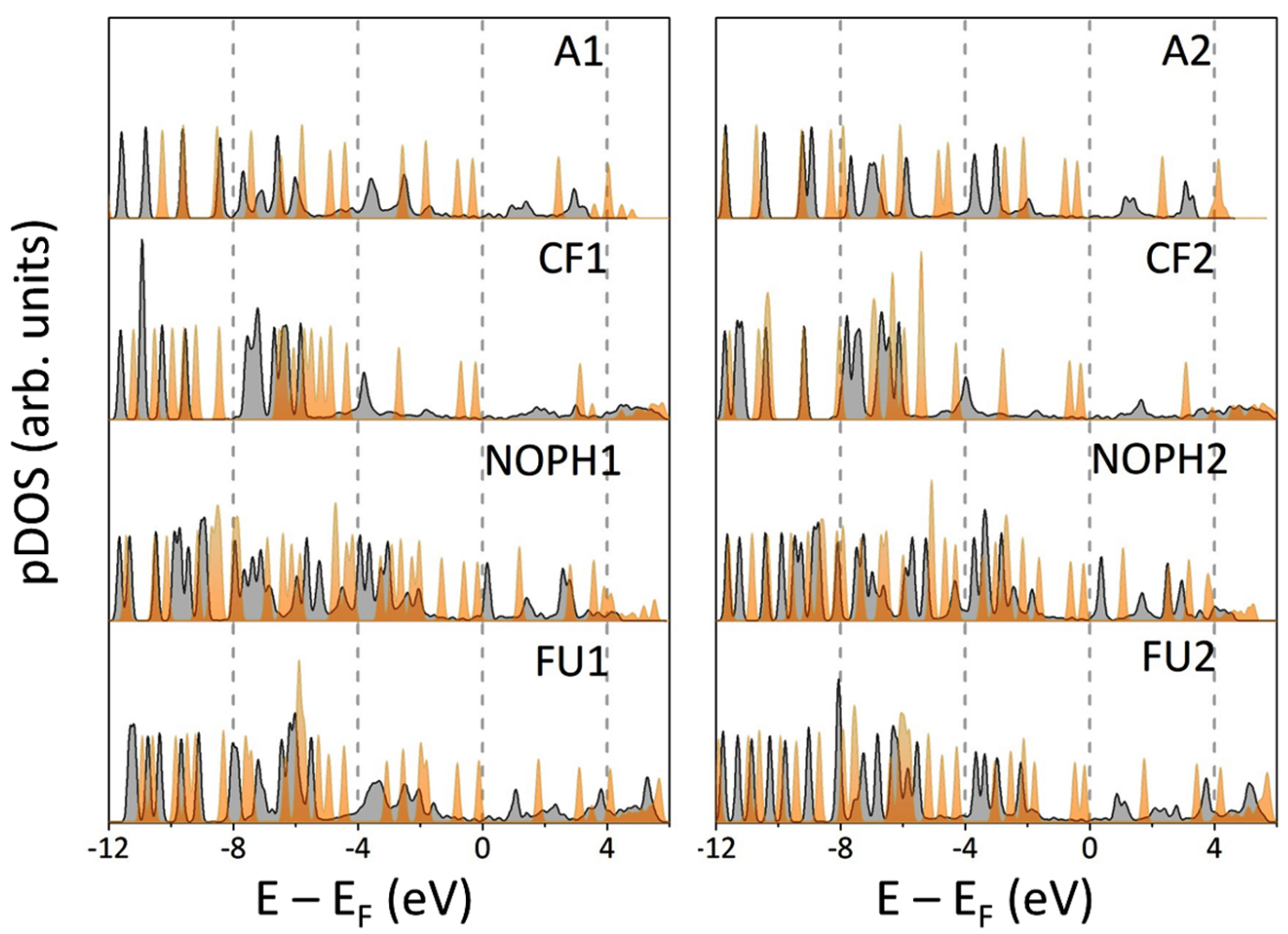

Figure 10: pDOS - Density of states projected over the atoms of the A, CF, NOPH, FU1 and FU2 derivatives. The gray shaded profile corresponds to the molecules adsorbed on the $\mathrm{Au}(111)$ surface, the non-interacting molecules are also included in the orange shaded profile. All the states has been described with respect to the Fermi level $\left(E_{F}\right)$

Further information on the nature of the molecule-metal interaction has been studied through the analysis of the Density of States projected over the atoms of the corresponding derivatives (pDOS). We show in Figure 10 the pDOS for A, CF, NOPH, and FU derivatives. The pDOS of the non-interacting system, which was obtained by placing the molecule far from the surface $(8 \AA)$, is also included. The low energy region in the pDOS, below $-12 \mathrm{eV}$, 
was discarded because it contains the inner bands (typically $\sigma$ molecular states). Those $\sigma$ states only exhibit a slight downshift in energy due to the molecule-metal charge transfer process. pDOS for the rest of derivatives can be found in the Supp.Info.

The main difference between the pDOS of the molecule far away and interacting with the surface lies in the loss of the well-defined peaks characteristic of the molecular states. The broadening of the molecular peaks is noticeable throughout the pDOS profile, especially above and below the Fermi level $\left(E_{F}\right)$. In the bonding region, a strong broadening is observed for those bands appearing at $\sim-4 \mathrm{eV}$, which is due to the interaction of the HOMO with the metal surface. Furthermore, the HOMO-surface interaction also demonstrates the relation between the bonding strength of the derivatives and its cis-trans configuration. Cisderivatives exhibit a stronger broadening and splitting in the bonding region, close to $-4 \mathrm{eV}$, upon adsorption. The pDOS peaks of the cis-derivatives also show a broadening below -4 $\mathrm{eV}$, although to a lesser extent. These features occur more weakly for the trans-derivatives. The only exception is shown by the CF derivatives, where CF2 displayed a broader peak than $\mathrm{CF} 1$ at $\sim-4 \mathrm{eV}$. The trends here observed denote a stronger molecule-metal interaction of the cis-derivatives (and the trans CF2) with the $\mathrm{Au}(111)$ surface, which agrees with the previous discussed results. In the region between -2 and $0 \mathrm{eV}$, all the derivatives exhibit the LUMO orbitals after been downshifted and becoming occupied states due to the $\mathrm{Au} \rightarrow \pi^{*}$ back-donation from the surface. Such changes in the molecular states upon adsorption are a well known phenomena which have been previously pointed out. ${ }^{83,92}$ The analysis of pDOS demonstrates that, despite having a larger Au-S distance, cis-derivatives strongly interact with the $\mathrm{Au}(111)$ surface. Therefore, these results emphasize our assumption that adsorption of these monosubstituted thiourea derivatives depends both on the Au-S chemical affinity and on the long range interactions between the functional groups and the $\mathrm{Au}(111)$ surface. 


\section{Non-Covalent Interactions}

According to the analysis of Figures 6 and 8 (see also related discussion), most likely long range interactions occur between different fragments of the thiourea derivatives and the $\mathrm{Au}(111)$ surface. Thus, in order to achieve a complete understanding of the molecule-metal interaction, we have used the Non-Covalent Interactions (NCI) approach ${ }^{52,93}$ to identify the long range interactions that can exist between the thiourea derivatives and the $\mathrm{Au}(111)$ surface.

Figure 11 displays the NCI mapping obtained by the representation of the reduced gradient density $(s)$ isosurfaces colored according to the corresponding values of $\operatorname{sign}\left(\lambda_{2} \rho\right)$. In all cases negative values of the $\operatorname{sign}\left(\lambda_{2} \rho\right)$ are observed indicating the presence of attractive forces between the derivatives and the $\mathrm{Au}(111)$ surface. As it was expected, there is a bonding interaction between the $\mathrm{S}$ atom in the molecule and the $\mathrm{Au}$ atoms of the surface. The isosurfaces deformation shows that in most cases the Au-S interaction can reach gold atoms far from the adsorption site, which is due to the highly polarizable electron density associated to the sulfur atom. Hydrogen bonding between the molecules and the $\mathrm{Au}(111)$ surface $(\mathrm{Au} \cdots \mathrm{N} 1-\mathrm{H})$ can be clearly distinguished from the NCI isosurfaces. In the case of cis-derivatives A1, PH1, CHPH1, FU3 and FU1, weak hydrogen bonds also appear in the regions corresponding to the functional group. Furthermore, these cis-derivatives show large regions of NCI, extended through the molecule-metal system. Such regions can be attributed to an interaction between the $\pi$ orbital of the molecule and the $d$ band of the $\mathrm{Au}(111)$ surface: $\pi-d_{\mathrm{Au}}$. On the other hand, the trans-derivatives FU2 and CF2 are the only cases where long range interaction with the $\mathrm{Au}(111)$ surface through the functional group have been observed. Small NCI isosurfaces in FU2 show interactions between the lone pair of the oxygen atom (furan group) and the $d$ band of the $\mathrm{Au}(111)$ surface $L P_{\mathrm{O}}-d_{\mathrm{Au}}$, while for CF2 the interaction occurs with the thiourea core and weakly with the fluorine atoms of the functional group.

All these features provide further explanation on the higher stability of cis-derivatives 


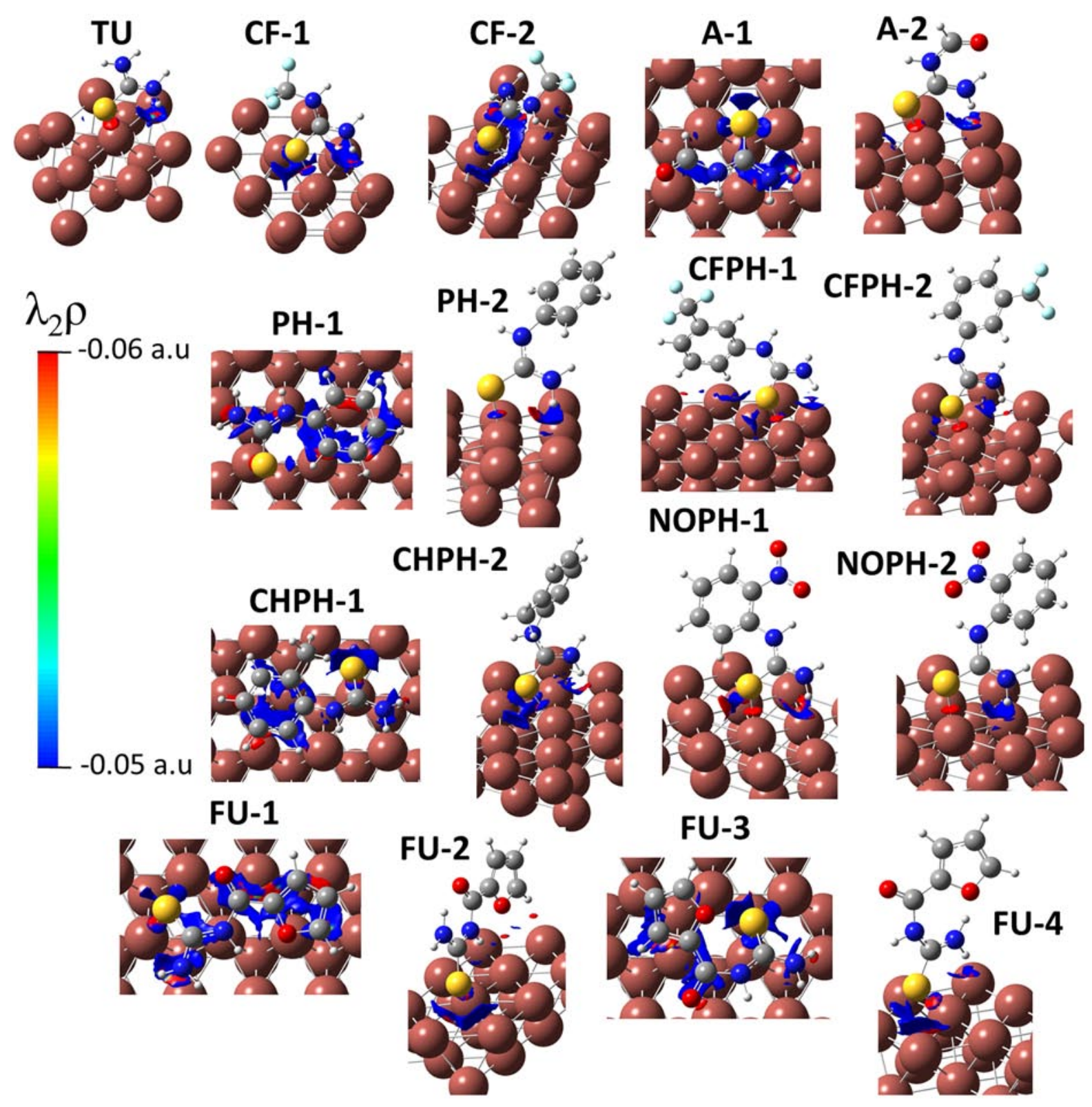

Figure 11: NCI isosurfaces representation for the reduced density gradient at the isovalue $s=0.3$ a.u. The color scale depicts the range of electron density taken in consideration $-0.06<\rho<-0.05$ a.u. For a better visualization of the isosurfaces the intermolecular NCI regions for all the molecules have been discarded. 
in comparison with the trans-derivatives, as well as on the anomalous behavior of the trans CF2 structure. The attractive NCI forces identified for the cis and trans CF2 derivatives, allow a stronger binding with the $\mathrm{Au}(111)$ surface enhancing the molecule-metal interaction. Therefore, the large Au-S distance observed in the cis and trans CF2 derivatives, does not mean a loss of stability; On the contrary, it reflects the equilibrium distance at which all the attractive forces are maximized. Nevertheless, the NCI forces appear due to the relative orientation of the molecules with respect to the $\mathrm{Au}(111)$ surface, which in turn is dictated by the Au-S interaction. The effective Au-S coupling remains to be the main force driving the adsorption of these monosubstituted thiourea derivatives, controlling the rest of interactions between the molecules and the $\mathrm{Au}(111)$ surface.

\section{Conclusions}

We have presented a systematic theoretical study, using a self-consistent periodic DFT method, identifying the interactions which drive the adsorption of several monosubstituted thiourea derivatives on the $\mathrm{Au}(111)$ surface. Two effects have to be considered to understand the nature of the molecule-metal interaction: the spatial configuration of the derivatives, cis or trans, and the electrondonating/withdrawing character of the functional group. The comparison of the non-substituted thiourea and the monosubstituted derivatives revealed a dependence between the cis-trans configuration and the adsorption geometries. Cis-derivatives showed geometries where the functional group was closer to the surface, specially the 2-furoyl and phenyl derivatives, adopting a lay-down arrangement. On the other hand, trans-derivatives exhibit adsorption geometries where the functional group was far from the surface. The analysis of the adsorption energy revealed that cis-derivatives bind more strongly to the surface than trans-derivatives. The higher stability of the cisderivatives was attributed to their lay-down arrangement which leads to an enhancement of the molecule-metal interaction through the coupling of $\pi$ electrons with the $\mathrm{Au}(111)$ surface. 
In addition, we have proved that the stability of molecule-metal system in the cis-derivatives also depended on the electrondonating/withdrawing nature of the functional group.

The characterization of molecule-metal interactions through the analysis of pDOS and the charge transfer allowed us to distinguish a $\pi \rightarrow \mathrm{Au}$ and $\mathrm{HOMO} \rightarrow \mathrm{Au}$ donation and a $\mathrm{Au}$ $\rightarrow \pi^{*}$ back-donation. The predominant electron donation in the total charge transfer process was the $\mathrm{HOMO} \rightarrow \mathrm{Au}$ which was affected by the electrondonating/withdrawing nature of the functional group of the derivatives. The fact that the charge transfer is modified by the different functional groups arises these monosubstituted thiourea derivatives as promising candidates in fields such as nanodevices or self-assembled, where tuning the electron transport is of paramount importance. ${ }^{94-97}$

This work also reveals that molecule-metal interactions can occur through one of the hydrogen atom of the unsubstituted nitrogen (N1) of the derivatives and the $\mathrm{Au}(111)$ surface, similarly to hydrogen bonding. The Au $\cdots \mathrm{H}-\mathrm{N} 1$ interaction appears due to the charge transfer that leads a negatively charged $\mathrm{Au}(111)$ surface allowing a $\mathrm{Au}^{\delta-} \mathrm{H}^{\delta+} \mathrm{N}^{\delta-}$ counterbalancing. Interestingly, this trend in the charge transfer produces an increment of the metal surface work function in all the derivatives. The ability to increase the working function of the metal and simultaneously generate a charge transfer towards the surface can be other of the potentialities of these derivatives.

Finally, the NCI plots give us further insight into the nature of the molecule-metal strength, allowing us to characterize $\pi$-Au and lone pair-Au as long range bonding interactions.

We consider that the results here presented provide a theoretical strategy to reveal the molecule-surface interactions, which in view of the growing interest in this type of system ${ }^{98-100}$ can be helpful to achieve a better understanding of the properties in hybrid organicmetal interfaces. The interest of such systems lies in their potential applications such as biosensors or atmospheric sensors. The design of such nanodevices would imply inclusion of solvent and environmental effects. This work opens the doors for future investigations in 
this direction.

\section{Acknowledgement}

The authors thank the generous allocation of computer time at Centro de Computación Científica at the Universidad Autónoma de Madrid (CCC-UAM). R.B. acknowledges Fundación Carolina at Spain, for a PhD fellowship. This work was partially supported by the "Proyecto Nacional de Ciencias Básicas", Cuba code P223LH001-079 "Absorción de moléculas organosulfuradas en superficies metálicas" and by the project CTQ2016-76061-P of the Spanish Ministry of Science and Innovation. S.D.T acknowledges financial support from the Spanish Ministry of Science and Innovation, through the "María de Maeztu" Programme for Units of Excellence in R\&D (CEX2018-000805-M). M. P. H. would like to thank to DGAPAUNAM for a 4-month fellowship through "Programa de Estancias de Investigación en la UNAM (PREI)".

\section{Supporting Information Available}

Molecular Electrostatic Potential and HOMO, LUMO, LUMO +1 molecular orbitals at the gas phase level of theory. Adsorption geometries. Charge density difference plots. Data of the transferred charge and energy of the HOMO level. Correlation between the work function variation and charge transfer. Projected Density of States. Planar cut and representation in the [111] direction of the charge density difference.

\section{References}

(1) Kislitsyn, D. A.; Taber, B. N.; Gervasi, C. F.; Mannsfeld, S. C. B.; Zhang, L.; Briseno, A. L.; Nazin, G. V. Coverage-Dependent Self-Assembly Regimes of Alkyl- 
Substituted Thiophene Oligomers on $\mathrm{Au}(111)$ : Scanning Tunneling Microscopy and Spectroscopy. J. Phys. Chem. C 2015, 119, 26959-26967.

(2) Bai, J.; Daaoub, A.; Sangtarash, S.; Li, X.; Tang, Y.; Zou, Q.; Sadeghi, H.; Liu, S.; Huang, X.; Tan, Z. et al. Anti-resonance features of destructive quantum interference in single-molecule thiophene junctions achieved by electrochemical gating. Nat. Mater. 2019, 18, 364-369.

(3) Benvidi, A.; Firouzabadi, A. D.; Tezerjani, M. D.; Moshtaghiun, S.; MazloumArdakani, M.; Ansarin, A. A highly sensitive and selective electrochemical DNA biosensor to diagnose breast cancer. J. Electroanal. Chem. 2015, 750, 57 - 64 .

(4) Wagner, M.; Qvortrup, K.; Grier, K. E.; Ottosen, M. R.; Petersen, J. O.; Tanner, D.; Ulstrup, J.; Zhang, J. Gold-carbonyl group interactions in the electrochemistry of anthraquinone thiols self-assembled on Au(111)-surfaces. Chem. Sci. 2019, 10, 39273936.

(5) Peiretti, L. F.; Quaino, P.; Tielens, F. Competition between Two High-Density Assemblies of Poly(phenyl)thiols on $\mathrm{Au}(111)$. J. Phys. Chem. C 2016, 120, 25462-25472.

(6) Bürgi, T. Properties of the gold-sulphur interface: from self-assembled monolayers to clusters. Nanoscale 2015, 7, 15553-15567.

(7) Gao, J.; Lin, H.; Qin, X.; Zhang, X.; Ding, H.; Wang, Y.; Rokni Fard, M.; Kaya, D.; Zhu, G.; Li, Q. et al. Probing Phase Evolutions of Au-Methyl-Propyl-Thiolate SelfAssembled Monolayers on $\mathrm{Au}(111)$ at the Molecular Level. J. Phys. Chem. B 2018, 122, 6666-6672.

(8) Rodríguez-Fernández, J.; Schmidt, S. B.; Lauritsen, J. V. Sulfur-driven switching of the Ullmann coupling on $\mathrm{Au}(111)$. Chem. Commun. 2018, 54, 3621-3624. 
(9) Mezour, M. A.; Perepichka, I. I.; Ivasenko, O.; Lennox, R. B.; Perepichka, D. F. Tridentate benzylthiols on $\mathrm{Au}(111)$ : control of self-assembly geometry. Nanoscale 2015, 7, 5014-5022.

(10) Giménez-Santamarina, S.; Cardona-Serra, S.; Gaita-Ariño, A. Theoretical insights on the importance of anchoring vs molecular geometry in magnetic molecules acting as junctions. J. Magn. Magn. Mater. 2019, 485, $212-216$.

(11) Komoto, Y.; Fujii, S.; Kiguchi, M. Single-molecule junctions of $\pi$ molecules. Mater. Chem. Front. 2018, 2, 214-218.

(12) Capitao, D.; Limoges, B.; Fave, C.; Schöllhorn, B. On the decisive role of the sulfurbased anchoring group in the electro-assisted formation of self-assembled monolayers on gold. Electrochim. Acta 2017, 257, 165 - 171.

(13) Engel, S.; Fritz, E.-C.; Ravoo, B. J. New trends in the functionalization of metallic gold: from organosulfur ligands to N-heterocyclic carbenes. Chem. Soc. Rev. 2017, 46, 2057-2075.

(14) Inkpen, M. S.; Liu, Z.-F.; Li, H.; Campos, L. M.; Neaton, J. B.; Venkataraman, L. Nonchemisorbed gold-sulfur binding prevails in self-assembled monolayers. Nat. Chem. 2019, 11, 351-358.

(15) Zugermeier, M.; Gruber, M.; Schmid, M.; Klein, B. P.; Ruppenthal, L.; Müller, P.; Einholz, R.; Hieringer, W.; Berndt, R.; Bettinger, H. F. et al. On-surface synthesis of heptacene and its interaction with a metal surface. Nanoscale 2017, 9, 12461-12469.

(16) Wilson, D.; de los Ángeles Arada, M.; Alegret, S.; del Valle, M. Lead(II) ion selective electrodes with PVC membranes based on two bis-thioureas as ionophores: 1,3bis(N'-benzoylthioureido)benzene and 1,3-bis(N'-furoylthioureido)benzene. J. Hazard. Mater. 2010, 181, $140-146$. 
(17) Semeniuc, R. F.; Reamer, T. J.; Blitz, J. P.; Wheeler, K. A.; Smith, M. D. Functionalized O-Alkyldithiocarbonates: A New Class of Ligands Designed for Luminescent Heterometallic Materials. Inorg. Chem. 2010, 49, 2624-2629.

(18) Saeed, A.; Flörke, U.; Erben, M. F. A review on the chemistry, coordination, structure and biological properties of 1-(acyl/aroyl)-3-(substituted) thioureas. J. Sulfur Chem. 2014, 35, 318-355.

(19) Limbut, W.; Kanatharana, P.; Mattiasson, B.; Asawatreratanakul, P.; Thavarungkul, P. A reusable capacitive immunosensor for carcinoembryonic antigen (CEA) detection using thiourea modified gold electrode. Anal. Chim. Acta 2006, $561,55-61$.

(20) Izaga, A.; Herrera, R. P.; Gimeno, M. C. Gold(I)-Mediated Thiourea Organocatalyst Activation: A Synergic Effect for Asymmetric Catalysis. Chem. Cat. Chem. 2017, 9, $1313-1321$.

(21) Weder, N.; Alberto, R.; Koitz, R. Thiourea Derivatives as Potent Inhibitors of Aluminum Corrosion: Atomic-Level Insight into Adsorption and Inhibition Mechanisms. J. Phys. Chem. C 2016, 120, 1770-1777.

(22) Wang, Y.; Hu, J.; Wang, Y.; Yu, L. A New Method for Preventing Corrosion Failure: Thiourea and Hexamethylenetetramine as Inhibitor for Copper. B. Korean Chem. Soc. 2016, 37, 1797-1811.

(23) Limbut, W.; Kanatharana, P.; Mattiasson, B.; Asawatreratanakul, P.; Thavarungkul, P. A comparative study of capacitive immunosensors based on selfassembled monolayers formed from thiourea, thioctic acid, and 3-mercaptopropionic acid. Biosens. Bioelectron. 2006, 22, $233-240$.

(24) Azzaroni, O.; Andreasen, G.; Blum, B.; Salvarezza, R. C.; Arvia, A. J. Scanning Tun- 
neling Microscopy Studies of the Electrochemical Reactivity of Thiourea on Au(111) Electrodes. J. Phys. Chem. B 2000, 104, 1395-1398.

(25) García, G.; Macagno, V. A.; Lacconi, G. I. Study of thiourea adsorption onto polycrystalline gold electrodes. Electrochim. Acta 2003, 48, 1273 - 1277, Microscopic and Nanoscopic Aspects of Corrosion and Corrosion Protection.

(26) Cheuquepán, W.; Pérez, J. M.; Orts, J. M.; Rodes, A. Spectroelectrochemical and DFT Study of Thiourea Adsorption on Gold Electrodes in Acid Media. J. Phys. Chem. C 2014, 118, 19070-19084.

(27) Cheuquepán, W.; Orts, J. M.; Rodes, A. On the electrochemical behavior of formamidine disulfide on gold electrodes in acid media. J. Electroanal. Chem. 2016, 764, 79 87.

(28) Hu, Z.-X.; Lan, H.; Ji, W. Role of the dispersion force in modeling the interfacial properties of molecule-metal interfaces: adsorption of thiophene on copper surfaces. Sci. Rep. 2014, 4, 5036.

(29) Romaner, L.; Nabok, D.; Puschnig, P.; Zojer, E.; Ambrosch-Draxl, C. Theoretical study of PTCDA adsorbed on the coinage metal surfaces, $\mathrm{Ag}(111), \mathrm{Au}(111)$ and Cu(111). New J. Phys. 2009, 11, 053010.

(30) Frisch, M. J.; Trucks, G. W.; Schlegel, H. B.; Scuseria, G. E.; Robb, M. A.; Cheeseman, J. R.; Scalmani, G.; Barone, V.; Petersson, G. A.; Nakatsuji, H. et al. Gaussian09 Revision E.01. 2009; Gaussian Inc. Wallingford CT.

(31) Kresse, G.; Hafner, J. Ab initio molecular dynamics for liquid metals. Phys. Rev. B. 1993, 47, 558-561.

(32) Kresse, G.; Hafner, J. Ab initio molecular-dynamics simulation of the liquid-metal- 
amorphous-semiconductor transition in germanium. Phys. Rev. B. 1994, 49, 1425114269.

(33) Kresse, G.; Furthmüller, J. Efficient iterative schemes for ab initio total-energy calculations using a plane-wave basis set. Phys. Rev. B. 1996, 54, 11169-11186.

(34) Kresse, G.; Furthmüller, J. Efficiency of ab-initio total energy calculations for metals and semiconductors using a plane-wave basis set. Comput. Mater. Sci. 1996, 6, 15-50.

(35) Zhao, Y.; Truhlar, D. G. The M06 suite of density functionals for main group thermochemistry, thermochemical kinetics, noncovalent interactions, excited states, and transition elements: two new functionals and systematic testing of four M06-class functionals and 12 other functionals. Theor. Chem. Acc. 2008, 120, 215-241.

(36) Perdew, J. P.; Chevary, J. A.; Vosko, S. H.; Jackson, K. A.; Pederson, M. R.; Singh, D. J.; Fiolhais, C. Atoms, molecules, solids, and surfaces: Applications of the generalized gradient approximation for exchange and correlation. Phys. Rev. B 1992, 46, 6671-6687.

(37) Perdew, J. P.; Chevary, J. A.; Vosko, S. H.; Jackson, K. A.; Pederson, M. R.; Singh, D. J.; Fiolhais, C. Erratum: Atoms, molecules, solids, and surfaces: Applications of the generalized gradient approximation for exchange and correlation. Phys. Rev. B 1993, 48, 4978-4978.

(38) Perdew, J. P.; Burke, K.; Ernzerhof, M. Generalized Gradient Approximation Made Simple. Phys. Rev. Lett. 1996, 77, 3865-3868.

(39) Perdew, J. P.; Burke, K.; Ernzerhof, M. Generalized Gradient Approximation Made Simple [Phys. Rev. Lett. 77, 3865 (1996)]. Phys. Rev. Lett. 1997, 78, 1396-1396.

(40) Blöch, P. E. Projector augmented-wave method. Phys. Rev. B. 1994, 50, 17953-17979. 
(41) Kresse, G.; Joubert, D. From ultrasoft pseudopotentials to the projector augmentedwave method. Phys. Rev. B. 1999, 59, 1758-1775.

(42) Methfessel, M.; Paxton, T. High-precision sampling for Brillouin-zone integration in metals. Phys. Rev. B. 1989, 40, 3616-3621.

(43) Freire, R. L. H.; Guedes-Sobrinho, D.; Kiejna, A.; Da Silva, J. L. F. Comparison of the Performance of van der Waals Dispersion Functionals in the Description of Water and Ethanol on Transition Metal Surfaces. J. Phys. Chem. C 2018, 122, 1577-1588.

(44) Steinmann, S. N.; Corminboeuf, C. Comprehensive Benchmarking of a DensityDependent Dispersion Correction. J. Chem. Theory Comput. 2011, 7, 3567-3577.

(45) Steinmann, S. N.; Corminboeuf, C. A generalized-gradient approximation exchange hole model for dispersion coefficients. J. Chem. Phys. 2011, 134, 044117.

(46) Brémond, É.; Golubev, N.; Steinmann, S. N.; Corminboeuf, C. How important is selfconsistency for the $\mathrm{dDsC}$ density dependent dispersion correction? J. Chem. Phys. 2014, 140, 18A516.

(47) Mete, E.; Yortanli, M.; Danişman, M. F. A van der Waals DFT study of chain length dependence of alkanethiol adsorption on $\mathrm{Au}(111)$ : physisorption vs. chemisorption. Phys. Chem. Chem. Phys. 2017, 19, 13756-13766.

(48) Gautier, S.; Steinmann, S. N.; Michel, C.; Fleurat-Lessard, P.; Sautet, P. Molecular adsorption at $\mathrm{Pt}(111)$. How accurate are DFT functionals? Phys. Chem. Chem. Phys. 2015, 17, 28921-28930.

(49) Grimme, S.; Antony, J.; Ehrlich, S.; Krieg, H. A consistent and accurate ab initio parametrization of density functional dispersion correction (DFT-D) for the 94 elements H-Pu. J. Chem. Phys. 2010, 132, 154104. 
(50) Grimme, S.; Ehrlich, S.; Goerigk, L. Effect of the damping function in dispersion corrected density functional theory. J. Comp. Chem. 2011, 32, 1456-1465.

(51) Contreras-García, J.; Johnson, E. R.; Keinan, S.; Chaudret, R.; Piquemal, J.-P.; Beratan, D. N.; Yang, W. NCIPLOT: A Program for Plotting Noncovalent Interaction Regions. J. Chem. Theory Comput. 2011, 7, 625-632.

(52) Johnson, E. R.; Keinan, S.; Mori-Sánchez, P.; Contreras-García, J.; Cohen, A. J.; Yang, W. Revealing Noncovalent Interactions. J. Am. Chem. Soc. 2010, 132, 64986506.

(53) Otero-de-la Roza, A.; Johnson, E. R.; Contreras-García, J. Revealing non-covalent interactions in solids: NCI plots revisited. Phys. Chem. Chem. Phys. 2012, 14, 1216512172.

(54) Otero-de-la Roza, A.; Blanco, M. A.; Pendás, A. M.; Luaña, V. Critic: a new program for the topological analysis of solid-state electron densities. Comput. Phys. Commun. $\mathbf{2 0 0 9 ,} 180,157-166$.

(55) Otero-de-la Roza, A.; Johnson, E. R.; Luaña, V. Critic2: A program for real-space analysis of quantum chemical interactions in solids. Comput. Phys. Commun. 2014, $185,1007-1018$.

(56) Bader, R. F. W. A quantum theory of molecular structure and its applications. Chem. Rev. 1991, 91, 893-928.

(57) Bader, R. F. W. Atoms in Molecules: A Quantum Theory; Oxford University Press, USA, 1994.

(58) Henkelman, G.; Arnaldsson, A.; Jonsson, H. A fast and robust algorithm for Bader decomposition of charge density. Comp. Mat. Sci. 2006, 36, $354-360$. 
(59) Sanville, E.; Kenny, S. D.; Smith, R.; Henkelman, G. Improved grid-based algorithm for Bader charge allocation. J. Comput. Chem. 2007, 28, 899-908.

(60) Tang, W.; Sanville, E.; Henkelman, G. A grid-based Bader analysis algorithm without lattice bias. J. Phys. Condens. Mat. 2009, 21, 084204.

(61) Santhanamoorthi, N.; Senthilkumar, K.; Kolandaivel, P. Absorption and emissionproperties of phenylene ethynylene oligomers: effect of substitution and $\pi$-conjugation length. Mol. Phys. 2009, 10\%, 1629-1639.

(62) Yamamoto, H.; Momiyama, N. Rich chemistry of nitroso compounds. Chem. Commun. 2005, 3514-3525.

(63) Cairo, R. R.; Stevens, A. M. P.; de Oliveira, T. D.; Batista, A. A.; Castellano, E. E.; Duque, J.; Soria, D. B.; Fantoni, A. C.; Corrêa, R. S.; Erben, M. F. Understanding the conformational changes and molecular structure of furoyl thioureas upon substitution. Spectrochim. Acta A 2017, 176, 8-17.

(64) Gil, D. M.; Lestard, M. D.; Estévez-Hernández, O.; Duque, J.; Reguera, E. Quantum chemical studies on molecular structure, spectroscopic (IR, Raman, UV-Vis), NBO and Homo-Lumo analysis of 1-benzyl-3-(2-furoyl) thiourea. Spectrochim. Acta A 2015, $145,553-562$.

(65) Bilić, A.; Reimers, J. R.; Hush, N. S. The structure, energetics, and nature of the chemical bonding of phenylthiol adsorbed on the $\mathrm{Au}(111)$ surface: Implications for densityfunctional calculations of molecular-electronic conduction. J. Chem. Phys. 2005, 122, 094708 .

(66) Cometto, F. P.; Paredes-Olivera, P.; Macagno, V. A.; Patrito, E. M. Density Functional Theory Study of the Adsorption of Alkanethiols on $\mathrm{Cu}(111), \mathrm{Ag}(111)$, and $\mathrm{Au}(111)$ in the Low and High Coverage Regimes. J. Phys. Chem. B 2005, 109, 2173721748. 
(67) Maksymovych, P.; Sorescu, D. C.; Yates, J. T. Methanethiolate Adsorption Site on Au(111): A Combined STM-DFT Study at the Single-Molecule Level. J. Phys. Chem. B 2006, 110, 21161-21167.

(68) Morokuma, K.; Pedersen, L.; Karplus, M. Structure of CH3 and CF3. J. Chem. Phys. 1968, 48, 4801-4802.

(69) Shahzad, N.; Nisa, R. U.; Ayub, K. Substituents effect on thermal electrocyclic reaction of dihydroazulene-vinylheptafulvene photoswitch: a DFT study to improve the photoswitch. Struct. Chem. 2013, 24, 2115-2126.

(70) Silva, A. L.; Freitas, V. L.; da Silva, M. D. R. Effects of methoxy and formyl substituents on the energetics and reactivity of alpha-naphthalenes: A calorimetric and computational study. Chemosphere 2014, 10\%, $203-210$.

(71) Shokri, A.; Wang, X.-B.; Kass, S. R. Electron-Withdrawing Trifluoromethyl Groups in Combination with Hydrogen Bonds in Polyols: Brønsted Acids, Hydrogen-Bond Catalysts, and Anion Receptors. J. Am. Chem. Soc. 2013, 135, 9525-9530.

(72) Chen, M.-W.; Ye, Z.-S.; Chen, Z.-P.; Wu, B.; Zhou, Y.-G. Enantioselective synthesis of trifluoromethyl substituted piperidines with multiple stereogenic centers via hydrogenation of pyridinium hydrochlorides. Org. Chem. Front. 2015, 2, 586-589.

(73) Safi, Z. S.; Omar, S. Proton affinity and molecular basicity of m- and p-substituted benzamides in gas phase and in solution: A theoretical study. Chem. Phys. Lett. 2014, $610-611,321-330$.

(74) Fertitta, E.; Voloshina, E.; Paulus, B. Adsorption of multivalent alkylthiols on Au(111) surface: Insights from DFT. J. Comput. Chem. 2014, 35, 204-213.

(75) Buimaga-Iarinca, L.; Morari, C. Adsorption of cysteine clusters on Au(110)-(1x1) surface: a DFT study. $R S C A d v$. 2013, 3, 5036-5044. 
(76) Awad, M. K. Semiempirical investigation of the inhibition efficiency of thiourea derivatives as corrosion inhibitors. J. Electroanal. Chem. 2004, 567, 219 - 225.

(77) Jaroschik, F. Picking One out of Three: Selective Single C-F Activation in Trifluoromethyl Groups. Chem. Eur. J. 2018, 24, 14572-14582.

(78) Gameel, K. M.; Sharafeldin, I. M.; Abourayya, A. U.; Biby, A. H.; Allam, N. K. Unveiling $\mathrm{CO}$ adsorption on $\mathrm{Cu}$ surfaces: new insights from molecular orbital principles. Phys. Chem. Chem. Phys. 2018, 20, 25892-25900.

(79) Figueiredo, M. C.; Hiltrop, D.; Sundararaman, R.; Schwarz, K. A.; Koper, M. T. Absence of diffuse double layer effect on the vibrational properties and oxidation of chemisorbed carbon monoxide on a Pt(111) electrode. Electrochim. Acta 2018, 281, $127-132$.

(80) Mehar, V.; Merte, L. R.; Choi, J.; Shipilin, M.; Lundgren, E.; Weaver, J. F. Adsorption of NO on FeOx Films Grown on $\mathrm{Ag}(111)$. J. Phys. Chem. C 2016, 120, 9282-9291.

(81) Zhang, L.; Filot, I. A.; Su, Y.-Q.; Liu, J.-X.; Hensen, E. J. Transition metal doping of $\operatorname{Pd}(111)$ for the $\mathrm{NO}+\mathrm{CO}$ reaction. J. Catal. 2018, 363, 154-163.

(82) Simpson, S.; Hooper, J.; Miller, D. P.; Kunkel, D. A.; Enders, A.; Zurek, E. Modulating Bond Lengths via Backdonation: A First-Principles Investigation of a Quinonoid Zwitterion Adsorbed to Coinage Metal Surfaces. J. Phys. Chem. C 2016, 120, 66336641.

(83) Hu, W.; Duan, S.; Zhang, Y.; Ren, H.; Jiang, J.; Luo, Y. Identifying the structure of 4chlorophenyl isocyanide adsorbed on $\mathrm{Au}(111)$ and $\mathrm{Pt}(111)$ surfaces by first-principles simulations of Raman spectra. Phys. Chem. Chem. Phys. 2017, 19, 32389-32397.

(84) Arefi, H. H.; Nolan, M.; Fagas, G. Role of the Head and/or Tail Groups of Adsorbed -[Xhead group]-Alkyl-[Xtail group] [X $=\mathrm{O}(\mathrm{H}), \mathrm{S}(\mathrm{H}), \mathrm{NH}(2)]$ Chains in Controlling 
the Work Function of the Functionalized H:Si(111) Surface. J. Phys. Chem. C 2015, $119,11588-11597$.

(85) Sauter, E.; Gilbert, C.-O.; Morin, J.-F.; Terfort, A.; Zharnikov, M. Mixed Monomolecular Films with Embedded Dipolar Groups on $\mathrm{Ag}(111)$. J. Phys. Chem. C 2018, 122, $19514-19523$.

(86) Roman, T.; Groß, A. Periodic Density-Functional Calculations on Work-Function Change Induced by Adsorption of Halogens on $\mathrm{Cu}(111)$. Phys. Rev. Lett. 2013, 110, 156804 .

(87) Bagus, P. S.; Käfer, D.; Witte, G.; Wöll, C. Work Function Changes Induced by Charged Adsorbates: Origin of the Polarity Asymmetry. Phys. Rev. Lett. 2008, 100, 126101.

(88) Leung, T. C.; Kao, C. L.; Su, W. S.; Feng, Y. J.; Chan, C. T. Relationship between surface dipole, work function and charge transfer: Some exceptions to an established rule. Phys. Rev. B 2003, 68, 195408.

(89) de Boer, B.; Hadipour, A.; Mandoc, M. M.; van Woudenbergh, T.; Blom, P. W. M. Tuning of Metal Work Functions with Self-Assembled Monolayers. Adv. Mater. 2005, $17,621-625$.

(90) Michaelides, A.; Hu, P.; Lee, M.-H.; Alavi, A.; King, D. A. Resolution of an Ancient Surface Science Anomaly: Work Function Change Induced by N Adsorption on W100. Phys. Rev. Lett. 2003, 90, 246103.

(91) Bagus, P. S.; Wöll, C.; Wieckowski, A. Dependence of surface properties on adsorbatesubstrate distance: Work function changes and binding energy shifts for I/Pt(111). Surf. Sci. 2009, 603, $273-283$. 
(92) Zhong, J.-H.; Jin, X.; Meng, L.; Wang, X.; Su, H.-S.; Yang, Z.-L.; Williams, C. T.; Ren, B. Probing the electronic and catalytic properties of a bimetallic surface with 3 nm resolution. Nat. Nanotechnol. 2017, 12, 132.

(93) Gavezzotti, A. Theoretical Aspects and Computer Modeling of the Molecular Solid State; Molecular Solid State; Wiley, 1997.

(94) Wang, S.; Zi, Y.; Zhou, Y. S.; Li, S.; Fan, F.; Lin, L.; Wang, Z. L. Molecular surface functionalization to enhance the power output of triboelectric nanogenerators. $J$. Mater. Chem. A 2016, 4, 3728-3734.

(95) Goldmann, C.; Lazzari, R.; Paquez, X.; Boissière, C.; Ribot, F.; Sanchez, C.; Chanéac, C.; Portehault, D. Charge Transfer at Hybrid Interfaces: Plasmonics of Aromatic Thiol-Capped Gold Nanoparticles. ACS Nano 2015, 9, 7572-7582.

(96) Singh, A.; Ansari, K.; Haque, J.; Dohare, P.; Lgaz, H.; Salghi, R.; Quraishi, M. Effect of electron donating functional groups on corrosion inhibition of mild steel in hydrochloric acid: Experimental and quantum chemical study. J. Taiwan Inst. Chem. E. 2018, 82, $233-251$.

(97) Abreu, D. S.; Temperini, M. L. A.; Abruña, H. D.; Diógenes, I. C. N. Modification of Gold's Work Function upon Adsorption of Mercaptobiphenylcarbonitrile: Experimental Evidence for a Theoretical Prediction. J. Phys. Chem. C 2018, 122, 6083-6092.

(98) Flores, M.; Cisternas, E.; Mella, A.; Jullian, D.; Nunez, A.; Soler, M. Adsorption of 2-thiophene curcuminoid molecules on a $\mathrm{Au}(111)$ surface. App. Surf. Sci. 2018, 427, $620-625$

(99) Jasper-Tönnies, T.; Poltavsky, I.; Ulrich, S.; Moje, T.; Tkatchenko, A.; Herges, R.; Berndt, R. Stability of functionalized platform molecules on $\mathrm{Au}(111)$. J. Chem. Phys. 2018, 149, 244705. 
(100) Farronato, M.; Bidermane, I.; Lüder, J.; Bouvet, M.; Vlad, A.; Jones, A.; Simbrunner, J.; Resel, R.; Brena, B.; Prévot, G. et al. New Quadratic Self-Assembly of DoubleDecker Phthalocyanine on Gold(111) Surface: From Macroscopic to Microscopic Scale. J. Phys. Chem. C 2018, 122, 26480-26488. 


\section{Graphical TOC Entry}

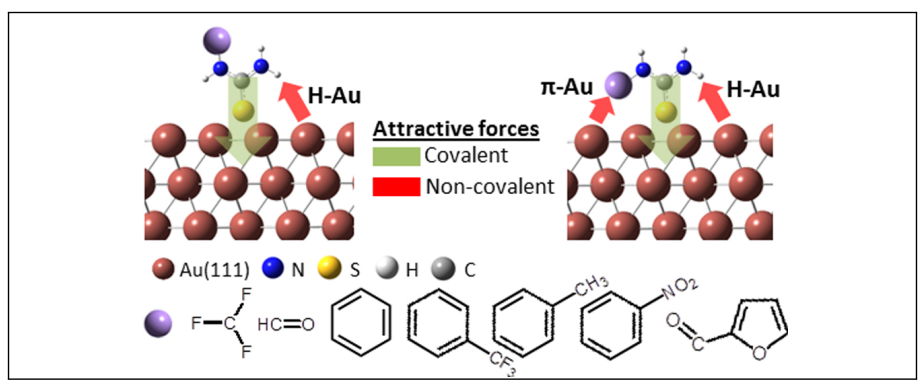

